\title{
Transition densities of spectrally positive Lévy processes
}

\author{
Lukasz Leżaj ${ }^{1}$ \\ Faculty of Pure and Applied Mathematics, Wrocław University of Science and Technology, \\ wyb. Wyspiańskiego 27, 50-370 Wrocław, Poland \\ (e-mail: lukasz.lezaj@pwr.edu.pl)
}

Received June 30, 2020; revised February 4, 2021

\begin{abstract}
We deduce the asymptotic behavior of transition densities for a large class of spectrally one-sided Lévy processes of unbounded variation satisfying mild condition imposed on the second derivative of the Laplace exponent or, equivalently, on the real part of the characteristic exponent. We also provide sharp two-sided estimates on the density when restricted additionally to processes without Gaussian component.
\end{abstract}

MSC: $60 \mathrm{~J} 35,60 \mathrm{~J} 75$

Keywords: spectrally one-sided Lévy process, heat kernel, Laplace exponent

\section{Introduction}

The aim of this paper is to discuss the behavior of the transition densities of spectrally one-sided Lévy processes of unbounded variation or, in other words, spectrally one-sided processes that are not subordinators with drift. Such processes, due to their specific structure, find natural applications in financial models, in particular, insurance risk modeling and queue theory, and therefore they have been intensively analyzed from that point of view. The prominent example here and, at the same time, one of the first questions we would like to ask in the financial setting is the so-called exit problem, the identification of the distribution of the pair $\left(\tau_{I}, X_{\tau_{I}}\right)$, where $I$ is an open interval, which has been intensively discussed over last decades. We refer to prominent works of Zolotarev [26], Takács [25], Emery [5], and Rogers [21]. Vast majority of results is expressed in terms of so-called scale functions, which have been of independent interest later on; see, for example, [10] or [16]. Also, specific structure of spectrally one-sided processes considerably simplifies the fluctuation theory, which for general Lévy processes is rather implicit. For details, we refer to books of Bertoin [1, Sects. VI, VII], Kyprianou [17], or Sato [23]. This short list is far from being complete, and for further discussion, we refer to the works mentioned and the references therein.

The abundant number of papers related to financial applications stays in stark contrast with the fact that surprisingly little is known about transition densities of general spectrally one-sided Lévy processes, although it seems that such knowledge can potentially be an important and useful tool. We may find, for example, asymptotic series expansion for the particular case of stable processes in the book of Zolotarev [27, Thm. 2.5.2] or the asymtotics with a fixed time variable under rather implicit assumptions in a recent article by Patie and

\footnotetext{
${ }^{1}$ The author was partially supported by the National Science Centre Poland: grant 2016/23/B/ST1/01665.
} 
Vaidyanathan [18], but to the author's best knowledge, general results are not obtained. Therefore the purpose of this paper is filling this gap in the theory and analysis of the behavior of the transition densities of spectrally one-sided Lévy processes in a feasibly wide generality.

Let us briefly describe our results. From theoretical point of view, the absence of negative (positive) jumps allows us to exploit techniques involving the Laplace transform, which can be easily proved to exist (see e.g., the book of Bertoin [1, Sect. VII]). We exploit that property in the first part, where we concentrate on the asymptotic behavior of the transition density, which is covered by Theorem 1 . Note that the result is very general, as the only assumption is the lower scaling property with index $\alpha>0$. In particular, we assume neither the upper scaling property nor the absolute continuity of the Lévy measure $\nu(\mathrm{d} x)$. Note that the independent sum of Brownian motion and a subordinator satisfying the scaling condition is admissible. The case $\alpha=2$ is also included. Recall that without Gaussian component, the condition of having unbounded variation is tantamount to satisfying the integral condition (see Preliminaries for elaboration)

$$
\int_{(0, \infty)}(1 \wedge x) \nu(\mathrm{d} x)=\infty .
$$

In fact, if this is the case, then the assumption $\alpha>0$ may seem superfluous at first sight, as the integrability condition (1.1), roughly speaking, requires enough singularity of order at least 1 . We may, however, construct a bit pathological example of a Lévy process of unbounded variation but with lower scaling index strictly smaller than 1. We refer the reader for details to Remark 1 and Example 2, but we highlight here that such processes are also included. Let us note in passing that, in some cases, it is easier to impose scaling condition on the real part of the characteristic exponent instead of on the second derivative of the Laplace exponent. These two are in fact equivalent, and we state that result in Corollary 6. If any of them is true, then by (4.5) and (4.6) we have, for some $x_{0} \geqslant 0$,

$$
x^{2} \varphi^{\prime \prime}(x) \approx \operatorname{Re} \psi(x), \quad x \geqslant x_{0} .
$$

Here $\varphi$ is the Laplace exponent, and $\psi$ is the characteristic exponent of Lévy process. Admittedly, one of the strengths of Theorem 1 lies in the fact that the expression in the exponent is given explicitly and there is no hidden unknown constant. Nonetheless, in view of the equation above, we can substitute the Laplace exponent with characteristic exponent, if necessary, at the cost of losing an exact formula and implicit constant, which will appear instead.

Next, we restrict ourselves to processes of unbounded variation without Gaussian component and focus on upper and lower estimates on the transition density. Whereas the former, covered by Theorem 2 , requires no additional assumptions and is independent of the previous results, the latter, consisting of Lemmas 3 and 4, requires apparently stronger conditions, $\alpha \geqslant 1$ and $\alpha>1$, respectively. They provide local and tail lower estimates on the transition density, and the proof of the latter relies strongly on Theorem 1 . As above, we point out that the condition $\alpha \geqslant 1$ is not very restrictive in the class of processes of unbounded variation. Finally, we merge all previous results in Theorem 4 to obtain sharp two-sided estimates. They require both lower and upper scaling condition with indices strictly separated from 1 and 2 , that is, $1<\alpha \leqslant \beta<2$. Let us note here that in contrast to symmetric processes, where a lot is already known, the general nonsymmetric case is still under development. It usually requires either implying familiar structure or imposing complex assumptions on the process; see, for example, $[8,12,13,14,15,19]$ and the references therein. Recently, estimates for subordinators in a general setting were obtained (see, e.g., [3, 4,7]). Still we are not aware of any paper that comprehensively treats the transition densities of general spectrally one-sided Lévy processes of unbounded variation. By inspecting the proofs of Lemmas 3 and 4, Theorem 3, and Proposition 9 we see that covering the limit cases using our methods is not possible, and it is not very surprising, as in general, they usually require more sophisticated methods or sometimes even a completely different approach. Nonetheless, the asymptotic behavior displayed by Theorem 1 covers both $\alpha=1$ and $\alpha=2$.

The paper is organized as follows. In Section 2, we introduce our setting and prove some basic properties of the Laplace exponent $\varphi$. Section 3 is devoted to the proof of the asymptotic behavior of the transition density. Upper and lower estimates are derived in Section 4, whereas in Section 5, we combine all previous results to obtain sharp two-sided estimates on the transition density. 


\section{Preliminaries}

Notation. Throughout the paper, $c, c_{1}, C_{1}, \ldots$ denote positive constants, which may vary from line to line. By $c=c(a)$ we mean that the constant $c$ depends only on one parameter $a$. For two functions $f, g:(0, \infty) \mapsto$ $[0, \infty]$, we write $f \approx g$ if the quotient $f / g$ stays between two positive constants. Analogous rule is applied to symbols $\lesssim$ and $\gtrsim$. We set $a \wedge b=\min \{a, b\}$ and $a \vee b=\max \{a, b\}$. The Borel sets on $\mathbb{R}$ are denoted by $\mathcal{B}(\mathbb{R})$.

Let $\mathbf{X}=\left(X_{t}: t \geqslant 0\right)$ be a spectrally positive Lévy process, that is, a Lévy process on $\mathbb{R}$ with only positive jumps that is not a subordinator with drift. There is a function $\psi: \mathbb{R} \mapsto \mathbb{C}$ such that for all $t>0$ and $\xi \in \mathbb{R}$,

$$
\mathbf{E} \mathrm{e}^{\mathrm{i} \xi X_{t}}=\mathrm{e}^{-t \psi(\xi)} .
$$

There are $\sigma \geqslant 0, b \in \mathbb{R}$, and $\sigma$-finite measure $\nu$ on $(0, \infty)$ satisfying $\int_{(0, \infty)}\left(1 \wedge x^{2}\right) \nu(\mathrm{d} x)<\infty$ such that for all $\xi \in \mathbb{R}$,

$$
\psi(\xi)=\sigma^{2} \xi^{2}-\mathrm{i} \xi b-\int_{(0, \infty)}\left(\mathrm{e}^{\mathrm{i} \xi x}-1-\mathrm{i} \xi x \mathbf{1}_{x<1}\right) \nu(\mathrm{d} x) .
$$

By $\varphi$ we denote the Laplace exponent of $\mathbf{X}$, that is,

$$
\mathbf{E e}^{-\lambda X_{t}}=\mathrm{e}^{t \varphi(\lambda)}, \quad \lambda \geqslant 0 .
$$

By holomorphic extension we can see that

$$
\varphi(\lambda)=\sigma^{2} \lambda^{2}-b \lambda+\int_{(0, \infty)}\left(\mathrm{e}^{-\lambda x}-1+\lambda x \mathbf{1}_{x<1}\right) \nu(\mathrm{d} x) .
$$

Let us introduce a symmetric, continuous, and nondecreasing majorant of $\operatorname{Re} \psi$,

$$
\psi^{*}(r)=\sup _{|z| \leqslant r} \operatorname{Re} \psi(z), \quad r>0
$$

and its generalized inverse function $\psi^{-1}(s)=\sup \left\{r>0: \psi^{*}(r)=s\right\}$. Then we have

$$
\psi^{*}\left(\psi^{-1}(s)\right)=s, \quad \psi^{-1}\left(\psi^{*}(s)\right) \geqslant s .
$$

Note that if $\int_{(0,1)} x \nu(\mathrm{d} x)<\infty$, then the underlying process has almost surely bounded variation, and we can rewrite (2.1) as follows:

$$
\psi(\xi)=\sigma^{2} \xi^{2}-\mathrm{i} \xi b_{0}-\int_{(0, \infty)}\left(\mathrm{e}^{\mathrm{i} \xi x}-1\right) \nu(\mathrm{d} x),
$$

where $b_{0}=b+\int_{(0,1)} x \nu(\mathrm{d} x)$. If this is the case, then $X_{t}=B_{t}+b_{0} t+T_{t}$, where $\mathbf{B}$ is a Brownian motion, and $\mathbf{T}=\left(T_{t}: t \geqslant 0\right)$ is a subordinator, that is, a one-dimensional Lévy process with nondecreasing paths that starts from 0 . Although it is usually clear from the setting, to avoid unnecessary confusions, we assume in the whole paper that $\mathbf{X}$ is of unbounded variation. By [23, Thms. 21.9, 24.10] this is true either when $\sigma>0$ or when the following condition is satisfied:

$$
\int_{(0,1)} x \nu(\mathrm{d} x)=\infty
$$


Therefore in this paper, we exclude the case where $\mathbf{X}$ is a subordinator with drift. However, the independent sum of Brownian motion and a subordinator is included. For results concerning heat kernel estimates for subordinators, we refer the reader to [7] and the references therein.

By differentiating (2.3) twice we can easily deduce that $\varphi$ is convex, and since $\mathbf{P}\left(X_{1} \leqslant-1\right)>0$ (in view of [23, Thm. 24.10]), we have $\varphi(\lambda) \rightarrow \infty$ as $\lambda \rightarrow \infty$. However, it is not necessarily positive. Indeed, differentiating (2.2) with respect to $\lambda$, setting $t=1$, and taking the limit as $\lambda \rightarrow 0^{+}$, we have

$$
\mathbf{E} X_{1}=-\varphi^{\prime}\left(0^{+}\right)=b+\int_{[1, \infty)} x \nu(\mathrm{d} x) .
$$

We see that $\mathbf{E} X_{1} \in(-\infty,+\infty]$. In particular, if $\mathbf{E} X_{1}>0$, then $\varphi<0$ in a neighborhood of the origin. Let $\theta_{0}$ be the largest root of $\varphi$. Similarly, let $\theta_{1}=\inf \left\{s>0: \varphi^{\prime}(s)>0\right\}$. We have $\theta_{1} \leqslant \theta_{0}$, and the equality may occur only for the case $\theta_{0}=\theta_{1}=0$. Note that there is always a root of $\varphi$ at $\lambda=0$ and, due to convexity of $\varphi$, at most one root for $\lambda>0$, precisely, in the case $\theta_{1}>0$. By the Wiener-Hopf factorization we get that $\varphi$ is necessarily of the form

$$
\varphi(\lambda)=\left(\lambda-\theta_{0}\right) \phi(\lambda)
$$

where $\phi$ is the Laplace exponent of a (possibly killed) subordinator, known as an ascending ladder height process, with the Lévy measure $\gamma$ given by

$$
\gamma((x, \infty))=\mathrm{e}^{\theta_{0}} x \int_{x}^{\infty} \mathrm{e}^{-\theta_{0} u} \nu((u, \infty)) \mathrm{d} u .
$$

See $\left[10\right.$, Sect. 4] and the references therein. It is known that $\phi$ is a Bernstein function, that is, a function in $C^{\infty}$ such that its derivative is completely monotone (see, e.g., [24] for a thorough analysis).

Following Pruitt [20], we define the concentration functions $K$ and $h$ by setting

$$
K(r)=\frac{\sigma^{2}}{r^{2}}+\frac{1}{r^{2}} \int_{(0, r)} s^{2} \nu(\mathrm{d} s), \quad r>0,
$$

and

$$
h(r)=\frac{\sigma^{2}}{r^{2}}+\int_{(0, \infty)}\left(1 \wedge \frac{s^{2}}{r^{2}}\right) \nu(\mathrm{d} s), \quad r>0 .
$$

Clearly, $h(r) \geqslant K(r)$. Note that by the Fubini-Tonelli theorem

$$
h(r)=2 \int_{r}^{\infty} K(s) s^{-1} \mathrm{~d} s .
$$

Furthermore, by [6, Lemma 4], for all $r>0$,

$$
\frac{1}{24} h\left(r^{-1}\right) \leqslant \psi^{*}(r) \leqslant 2 h\left(r^{-1}\right)
$$

Moreover, if the Lévy measure $\nu(\mathrm{d} x)$ has a monotone density $\nu(x)$, then obviously, for any $r>0$,

$$
r \nu(r) \leqslant K(r) \leqslant h(r) .
$$


Let us introduce the notions of almost monotonicity and scaling property, which are ubiquitous in the paper. First, a function $f:\left[x_{0}, \infty\right) \mapsto[0, \infty)$ is almost increasing on $\left(x_{1}, \infty\right)$ for some $x_{1} \geqslant x_{0}$ if there is $c \in(0,1]$ such that for all $y \geqslant x>x_{1}, f(y) \geqslant c f(x)$. Similarly, it is almost decreasing on $\left(x_{1}, \infty\right)$ if there is $C \geqslant 1$ such that for all $y \geqslant x>x_{1}, f(y) \leqslant C f(x)$.

We say that a function $f:\left[x_{0}, \infty\right) \mapsto[0, \infty)$ has weak lower scaling property at infinity if there are $\alpha \in$ $\mathbb{R}, c \in(0,1]$, and $x_{1} \geqslant x_{0}$ such that for all $\lambda \geqslant 1$ and $x>x_{1}, f(\lambda x) \geqslant c \lambda^{\alpha} f(x)$. In such case, we write $f \in \mathrm{WLSC}\left(\alpha, c, x_{1}\right)$. Analogously, we say that $f$ has a weak upper scaling property at infinity $(f \in$ $\left.\operatorname{WUSC}\left(\beta, C, x_{1}\right)\right)$ if there are $\beta \in \mathbb{R}, C \geqslant 1$, and $x_{1} \geqslant x_{0}$ such that for all $\lambda \geqslant 1$ and $x>x_{1}$,

$$
f(\lambda x) \leqslant C \lambda^{\beta} f(x) .
$$

A convenient equivalent definition of scaling property is provided in [2]. Namely, $f \in \operatorname{WLSC}\left(\alpha, c, x_{1}\right)$ if and only if the function $\left(x_{1}, \infty\right) \ni x \mapsto f(x) x^{-\alpha}$ is almost increasing. Analogously, $f \in \operatorname{WUSC}\left(\beta, C, x_{1}\right)$ if and only if the function $\left(x_{1}, \infty\right) \ni x \mapsto f(x) x^{-\beta}$ is almost decreasing. Finally, a function $f:\left[x_{0}, \infty\right) \mapsto[0, \infty)$ has a doubling property on $\left(x_{1}, \infty\right)$ if there are $x_{1} \geqslant x_{0}$ and $C \geqslant 1$ such that for all $x>x_{1}$,

$$
C^{-1} f(x) \leqslant f(2 x) \leqslant C f(x)
$$

Note here that a nonincreasing function with weak lower scaling property and nondecreasing function with weak upper scaling property have a doubling property. In particular, $\varphi^{\prime \prime} \in \operatorname{WLSC}\left(\alpha-2, c, x_{0}\right)$ has a doubling property.

\subsection{Properties of the Laplace exponent $\varphi$}

First, note that by differentiating (2.3) and using the inequality $x \mathrm{e}^{-x} \leqslant 1-\mathrm{e}^{-x}, x>0$, we get that for all $\lambda \geqslant 0$,

$$
\varphi(\lambda) \leqslant \lambda \varphi^{\prime}(\lambda)
$$

Furthermore, if $\theta_{0}>0$, then $-\varphi$ is positive and concave on $\left(0, \theta_{1}\right)$. Thus, for all $x \leqslant \theta_{1}$ and $\lambda \leqslant 1$,

$$
-\varphi(x)-(-\varphi(\lambda x)) \leqslant(1-\lambda) x\left(-\varphi^{\prime}(\lambda x)\right) .
$$

Thus, by (2.9), for all $x \leqslant \theta_{1}$ and $\lambda \leqslant 1$,

$$
\lambda(-\varphi(x)) \leqslant-\varphi(\lambda x) .
$$

Proposition 1. There are $C_{1}, C_{2} \geqslant 1$ such that $\varphi^{\prime} \in \operatorname{WUSC}\left(1, C_{1}, 2 \theta_{1} \wedge \theta_{0}\right)$ and $\varphi \in \operatorname{WUSC}\left(2, C_{2}, 2 \theta_{0}\right)$. Furthermore, if $\theta_{0}=0$ and $\varphi^{\prime}(0)=0$, then $C_{1}=C_{2}=1$, that is. for all $x>0$ and $\lambda \geqslant 1$,

$$
\varphi^{\prime}(\lambda x) \leqslant \lambda \varphi^{\prime}(x) \quad \text { and } \quad \varphi(\lambda x) \leqslant \lambda^{2} \varphi(x) .
$$

Proof. Let $\lambda \geqslant 1$. First, note that by the monotonicity of $\varphi^{\prime \prime}$, for all $x>\theta_{1}$,

$$
\varphi^{\prime}(\lambda x)-\varphi^{\prime}\left(\lambda \theta_{1}\right)=\int_{\lambda \theta_{1}}^{\lambda x} \varphi^{\prime \prime}(s) \mathrm{d} s=\lambda \int_{\theta_{1}}^{x} \varphi^{\prime \prime}(\lambda s) \mathrm{d} s \leqslant \lambda \int_{\theta_{1}}^{x} \varphi^{\prime \prime}(s) \mathrm{d} s=\lambda\left(\varphi^{\prime}(x)-\varphi^{\prime}\left(\theta_{1}\right)\right) .
$$

Thus we get the claim for $\varphi^{\prime}$ in the case $\theta_{1}=0$ and $\varphi^{\prime}(0)=0$. If $\theta_{1}=0$ but $\varphi^{\prime}(0)>0$, then we clearly have $\varphi^{\prime}(0) \leqslant \lambda \varphi^{\prime}(x)$ for all $x>0$ and $\lambda \geqslant 1$, and we get the claim with $C_{1}=2$. Finally, if $\theta_{1}>0$, then it remains to prove that there is $c>0$ such that for all $x>2 \theta_{1} \wedge \theta_{0}$ and $\lambda \geqslant 1$,

$$
\varphi^{\prime}\left(\lambda \theta_{1}\right) \leqslant c \lambda \varphi^{\prime}(x) .
$$


Since $\varphi^{\prime}\left(\theta_{1}\right)=0$, by the monotonicity of $\varphi^{\prime \prime}$ and $\varphi^{\prime}$ we obtain

$$
\varphi^{\prime}\left(\lambda \theta_{1}\right)=\int_{\theta_{1}}^{\lambda \theta_{1}} \varphi^{\prime \prime}(s) \mathrm{d} s \leqslant(\lambda-1) \theta_{1} \varphi^{\prime \prime}\left(\theta_{1}\right) \leqslant \lambda \theta_{1} \varphi^{\prime \prime}\left(\theta_{1}\right) \leqslant c \lambda \varphi^{\prime}(x),
$$

where $c=\left(\theta_{1} \varphi^{\prime \prime}\left(\theta_{1}\right)\right) / \varphi^{\prime}\left(2 \theta_{1} \wedge \theta_{0}\right)$, and (2.11) follows. Now, with the first part proved, a similar argument applies to the second and therefore is omitted.

Proposition 2. There is $C=C(\varphi) \geqslant 1$ such that for all $x>2 \theta_{0}$, we have

$$
\varphi(x) \leqslant x \varphi^{\prime}(x) \leqslant C \varphi(x) .
$$

Furthermore, for all $x>2 \theta_{1}$,

$$
2 \varphi^{\prime}(x) \geqslant x \varphi^{\prime \prime}(x)
$$

Proof. We first note that the first inequality of (2.12) follows from (2.9). Let $x>2 \theta_{0}$ and $1 \leqslant b<a$. By the monotonicity of $\varphi^{\prime}$ we have $\varphi(a x)-\varphi(b x) \geqslant x(a-b) \varphi^{\prime}(b x)$. Put $b=1$ and $a=2$. By Proposition 1 we have

$$
\frac{x \varphi^{\prime}(x)}{\varphi(x)} \leqslant \frac{\varphi(2 x)}{\varphi(x)}-1 \leqslant 4 \tilde{C}-1,
$$

where $\tilde{C}$ is taken from Proposition 1, and the first part follows. For the proof of the second part, it remains to note that by the monotonicity of $\varphi^{\prime \prime}$, for $x>2 \theta_{1}$ we have

$$
\frac{\varphi^{\prime}(x)}{x \varphi^{\prime \prime}(x)} \geqslant \frac{\varphi^{\prime}(x)-\varphi^{\prime}\left(\theta_{1}\right)}{x \varphi^{\prime \prime}(x)}=\frac{1}{x} \int_{\theta_{1}}^{x} \frac{\varphi^{\prime \prime}(s)}{\varphi^{\prime \prime}(x)} \mathrm{d} s \geqslant 1-\frac{\theta_{1}}{x} .
$$

Thus for $x>2 \theta_{1}$, we get the claim.

Corollary 1. There is $c=c(\varphi)>0$ such that for all $x \in\left(0, \theta_{0} / 2\right) \cup\left(2 \theta_{0}, \infty\right)$,

$$
|\varphi(x)| \geqslant c x^{2} \varphi^{\prime \prime}(x) \text {. }
$$

The implied constant $c$ depends only on $\theta_{0}$.

Proof. In view of Proposition 2, it remains to prove that if $\theta_{0}>0$, then there is $c>0$ such that for all $x<\theta_{0} / 2$,

$$
-\varphi(x) \geqslant c x^{2} \varphi^{\prime \prime}(x)
$$

From (2.6) we have $\varphi^{\prime \prime}(x)=2 \phi^{\prime}(x)+\left(\theta_{0}-x\right)\left(-\phi^{\prime \prime}(x)\right)$. By [11, Lemma 3.9.34], for any $n \in \mathbb{N}_{+}$,

$$
\phi(\lambda) \geqslant \frac{(-1)^{n+1}}{n !} \lambda^{n} \phi^{(n)}(\lambda), \quad \lambda>0 .
$$

Hence, for $x<\theta_{0} / 2$,

$$
-\varphi(x)=\left(\theta_{0}-x\right) \phi(x) \gtrsim \phi(x) \geqslant x \phi^{\prime}(x) \gtrsim x^{2} \phi^{\prime}(x) .
$$

Moreover, $\left(\theta_{0}-x\right) \phi(x) \gtrsim\left(\theta_{0}-x\right) x^{2}\left(-\phi^{\prime \prime}(x)\right)$, which, together with (2.14), implies (2.13), and the claim follows. 
Now we deduce some properties of $\varphi$ and its derivatives following from scaling properties.

Proposition 3. Suppose $\varphi^{\prime \prime} \in \mathrm{WLSC}\left(\alpha-2, c, x_{0}\right)$ for some $c \in(0,1], x_{0} \geqslant 0$, and $\alpha>0$. Assume that $\varphi^{\prime}\left(\theta_{1}\right)=0$. Then $\varphi^{\prime} \in \mathrm{WLSC}\left(\alpha-1, c, x_{0} \vee \theta_{1}\right)$ and $\varphi \in \mathrm{WLSC}\left(\alpha, c, x_{0} \vee \theta_{0}\right)$.

Proof. We proceed as in the proof of Proposition 1. Let $\lambda \geqslant 1$ and $x>\theta_{1} \vee x_{0}$. By the weak scaling property of $\varphi^{\prime \prime}$ we have

$$
\begin{aligned}
\varphi^{\prime}(\lambda x) & \geqslant \varphi^{\prime}(\lambda x)-\varphi^{\prime}\left(\lambda\left(\theta_{1} \vee x_{0}\right)\right)=\int_{\lambda\left(\theta_{1} \vee x_{0}\right)}^{\lambda x} \varphi^{\prime \prime}(s) \mathrm{d} s=\lambda \int_{\theta_{1} \vee x_{0}}^{x} \varphi^{\prime \prime}(\lambda s) \mathrm{d} s \\
& \geqslant c \lambda^{\alpha-1} \int_{\theta_{1} \vee x_{0}}^{x} \varphi^{\prime \prime}(s) \mathrm{d} s=c \lambda^{\alpha-1}\left(\varphi^{\prime}(x)-\varphi^{\prime}\left(\theta_{1} \vee x_{0}\right)\right),
\end{aligned}
$$

and the claim follows for the case $\theta_{1} \geqslant x_{0}$. If $\theta_{1}<x_{0}$, then by differentiating (2.3) we conclude that $\varphi^{\prime}(\lambda) \rightarrow \infty$ as $\lambda \rightarrow \infty$. Since it is also monotone, there is $x_{1}>x_{0}$ such that $\varphi^{\prime}(x) \geqslant 2 \varphi^{\prime}\left(x_{0}\right)$ for all $x>x_{1}$, and, consequently, $\varphi^{\prime} \in \operatorname{WLSC}\left(\alpha-1, \tilde{c}, x_{1}\right)$ for some $\tilde{c} \in(0,1]$. Finally, using the continuity and positivity of $\varphi^{\prime}$, we can extend the scaling area to $\left(x_{0}, \infty\right)$ at the expense of worsening the constant. The proof of the weak scaling property of $\varphi$ follows by an analogous argument.

Proposition 4. Let $\varphi$ be the Laplace exponent of a spectrally positive Lévy process of infinite variation such that $\varphi^{\prime}\left(\theta_{1}\right)=0$. Then $\varphi^{\prime} \in \operatorname{WLSC}\left(\tau, c, x_{0}\right)$ for some $c \in(0,1], x_{0} \geqslant \theta_{1}$, and $\tau>0$ if and only if $\varphi^{\prime \prime} \in \operatorname{WLSC}\left(\tau-1, c^{\prime}, x_{1}\right)$ for some $c^{\prime} \in(0,1]$ and $x_{1} \geqslant 0$. The first condition implies the latter with $x_{1}=x_{0}$, while the latter yields the first with $x_{0}=x_{1} \vee \theta_{1}$. Furthermore, if $\varphi^{\prime} \in \operatorname{WLSC}\left(\tau, c, x_{0}\right)$, then there is $C \geqslant 1$ such that for all $x>x_{0} \vee 2 \theta_{1}$,

$$
C^{-1} \varphi^{\prime}(x) \leqslant x \varphi^{\prime \prime}(x) \leqslant C \varphi^{\prime}(x) .
$$

Proof. Assume first that $\varphi^{\prime \prime} \in \operatorname{WLSC}\left(\tau-1, c^{\prime}, x_{1}\right)$. We claim that (2.15) holds. In view of Proposition 2, it suffices to prove the first inequality. First, let $x>2 \theta_{1} \vee x_{1}$. By the weak scaling property of $\varphi^{\prime \prime}$ we have

$$
\frac{\varphi^{\prime}(x)-\varphi^{\prime}\left(\theta_{1} \vee x_{1}\right)}{x \varphi^{\prime \prime}(x)}=\frac{1}{x} \int_{\theta_{1} \vee x_{1}}^{x} \frac{\varphi^{\prime \prime}(s)}{\varphi^{\prime \prime}(x)} \mathrm{d} s \leqslant \frac{1}{c^{\prime} x^{\tau}} \int_{\theta_{1} \vee x_{1}}^{x} s^{\tau-1} \mathrm{~d} s \leqslant \frac{1}{c^{\prime} \tau}
$$

Thus we get (2.15) if $x_{1} \leqslant \theta_{1}$. If this is not the case, note that since $\varphi^{\prime \prime} \in \operatorname{WLSC}\left(\tau-1, c, x_{1}\right)$ and $\tau>0$, the function $\left(x_{1}, \infty\right) \ni x \mapsto x \varphi^{\prime \prime}(x)$ is almost increasing. Thus for $x>2 x_{1}>0, x \varphi^{\prime \prime}(x) \geqslant c 2 x_{1} \varphi^{\prime \prime}\left(2 x_{1}\right)$. Since $\varphi^{\prime \prime}$ is continuous and positive on $\left[x_{1}, 2 x_{1}\right]$, we get that $x \varphi^{\prime \prime}(x) \gtrsim 1$ for all $x>x_{1}$, and (2.15) follows. The scaling property of $\varphi^{\prime}$ is now an immediate consequence.

Now assume that $\varphi^{\prime} \in \operatorname{WLSC}\left(\tau, c, x_{0}\right)$. By the monotonicity of $\varphi^{\prime \prime}$, for $0<b<a$,

$$
\frac{\varphi^{\prime}(a x)-\varphi^{\prime}(b x)}{\varphi^{\prime}(x)} \leqslant \frac{x(a-b) \varphi^{\prime \prime}(b x)}{\varphi^{\prime}(x)} .
$$

Put $b=1$. Then by the scaling property of $\varphi^{\prime}$ we have

$$
\frac{x(a-1) \varphi^{\prime \prime}(x)}{\varphi^{\prime}(x)} \geqslant \frac{\varphi^{\prime}(a x)}{\varphi^{\prime}(x)}-1 \geqslant c a^{\tau}-1
$$

for all $x>x_{0}$. Thus for $a=2^{1 / \tau} c^{-1 / \tau}$, we obtain that $\varphi^{\prime}(x) \lesssim x \varphi^{\prime \prime}(x)$ for all $x>x_{0}$, which, combined with Proposition 2, yields (2.15), and the scaling property of $\varphi^{\prime \prime}$ follows. This completes the proof. 
Combining Propositions 2, and 4, we immediately obtain the following corollary.

Corollary 2. Let $\varphi$ be the Laplace exponent of a spectrally positive Lévy process of infinite variation such that $\varphi^{\prime}\left(\theta_{1}\right)=0$. Then $\varphi \in \operatorname{WLSC}\left(\alpha, c, x_{0}\right)$ for some $c \in(0,1], x_{0} \geqslant \theta_{0}$, and $\alpha>1$ if and only if $\varphi^{\prime \prime} \in \operatorname{WLSC}\left(\alpha-2, c^{\prime}, x_{1}\right)$ for some $c^{\prime} \in(0,1]$ and $x_{1} \geqslant 0$. The first condition implies the latter with $x_{1}=x_{0}$, while the latter yields the first with $x_{0}=x_{1} \vee \theta_{0}$. Furthermore, if $\varphi \in \operatorname{WLSC}\left(\alpha, c, x_{0}\right)$, then there is $C \geqslant 1$ such that for all $x>x_{0} \vee 2 \theta_{0}$,

$$
C^{\prime-1} \varphi(x) \leqslant x^{2} \varphi^{\prime \prime}(x) \leqslant C^{\prime} \varphi(x) .
$$

Lemma 1. Suppose $\varphi^{\prime \prime} \in \operatorname{WLSC}\left(\alpha-2, c, x_{0}\right)$ for some $c \in(0,1], x_{0} \geqslant 0$, and $\alpha>0$. There is a constant $C>0$ such that for all $x>x_{0}$,

$$
C \varphi^{\prime \prime}(x) \leqslant \sigma^{2}+\int_{(0,1 / x)} s^{2} \nu(\mathrm{d} s)
$$

Proof. First, assume that $\sigma=0$; the extension to any $\sigma$ is immediate. Let $f:(0, \infty) \mapsto \mathbb{R}$ be the function defined as

$$
f(t)=\int_{(0, t)} s^{2} \nu(\mathrm{d} s)
$$

Note that by the Fubini-Tonelli theorem, for $x>0$, we have

$$
\mathcal{L} f(x)=\int_{0}^{\infty} \mathrm{e}^{-x t} \int_{(0, t)} s^{2} \nu(\mathrm{d} s) \mathrm{d} t=\int_{(0, \infty)} s^{2} \int_{s}^{\infty} \mathrm{e}^{-x t} \mathrm{~d} t \nu(\mathrm{d} s)=x^{-1} \varphi^{\prime \prime}(x) .
$$

Since $f$ is nondecreasing, for any $s>0$,

$$
\varphi^{\prime \prime}(x)=x \mathcal{L} f(x) \geqslant \int_{s}^{\infty} \mathrm{e}^{-t} f\left(\frac{t}{x}\right) \mathrm{d} t \geqslant \mathrm{e}^{-s} f\left(\frac{s}{x}\right) .
$$

Hence for any $u>2$,

$$
\varphi^{\prime \prime}(x)=\int_{0}^{u} \mathrm{e}^{-s} f\left(\frac{x}{s}\right) \mathrm{d} s+\int_{u}^{\infty} \mathrm{e}^{-s} f\left(\frac{x}{s}\right) \mathrm{d} s \leqslant f\left(\frac{u}{x}\right)+\int_{u}^{\infty} \mathrm{e}^{-s / 2} \varphi^{\prime \prime}\left(\frac{x}{2}\right) \mathrm{d} s .
$$

Therefore setting $x=\lambda u>2 x_{0}$, by the weak scaling property of $\varphi^{\prime \prime}$ we have

$$
f\left(\frac{1}{\lambda}\right) \geqslant \varphi^{\prime \prime}(u \lambda)-2 \mathrm{e}^{-u / 2} \varphi^{\prime \prime}\left(\frac{u \lambda}{2}\right) \geqslant\left(2^{\alpha-2} c-2 \mathrm{e}^{-u / 2}\right) \varphi^{\prime \prime}\left(\frac{u \lambda}{2}\right) .
$$

At this stage, we choose $u>2$ such that $2^{\alpha-2} c-2 \mathrm{e}^{-u / 2} \geqslant 2^{-2} c$. Then again, by the weak scaling property of $\varphi^{\prime \prime}$, for $\lambda>x_{0}$,

$$
f\left(\frac{1}{\lambda}\right) \geqslant c 2^{-2} \varphi^{\prime \prime}\left(\frac{u \lambda}{2}\right) \geqslant\left(\frac{c}{u}\right)^{2} \varphi^{\prime \prime}(\lambda),
$$

which ends the proof. 
Since

$$
K\left(\frac{1}{x}\right) \leqslant \mathrm{ex}^{2} \varphi^{\prime \prime}(x)
$$

by Lemma 1 we immediately obtain the following corollary.

Corollary 3. Suppose $\varphi^{\prime \prime} \in \operatorname{WLSC}\left(\alpha-2, c, x_{0}\right)$ for some $c \in(0,1], x_{0} \geqslant 0$, and $\alpha>0$. Then there is $C \geqslant 1$ such that for all $x>x_{0}$,

$$
C x^{2} \varphi^{\prime \prime}(x) \leqslant K\left(\frac{1}{x}\right) \leqslant \mathrm{e}^{2} \varphi^{\prime \prime}(x)
$$

Before embarking on our main results, let us prove a key lemma, which provides control on the real part of the holomorphic extension of the Laplace exponent.

Lemma 2. Suppose that $\varphi^{\prime \prime} \in \operatorname{WLSC}\left(\alpha-2, c, x_{0}\right)$ for some $c \in(0,1], x_{0} \geqslant 0$, and $\alpha>0$. Then there exists $C>0$ such that for all $w>x_{0}$ and $\lambda \in \mathbb{R}$,

$$
\operatorname{Re}(\varphi(w)-\varphi(w+\mathrm{i} \lambda)) \geqslant C \lambda^{2}\left(\varphi^{\prime \prime}(|\lambda| \vee w)\right) .
$$

Proof. By the integral representation (2.3), for $\lambda \in \mathbb{R}$, we have

$$
\operatorname{Re}(\varphi(w)-\varphi(w+\mathrm{i} \lambda))=\sigma^{2} \lambda^{2}+\int_{(0, \infty)}(1-\cos \lambda s) \mathrm{e}^{-w s} \nu(\mathrm{d} s) .
$$

In particular, we see that the expression above is symmetric in $\lambda$. Thus it suffices to consider $\lambda>0$. Moreover, we infer that

$$
\operatorname{Re}(\varphi(w)-\varphi(w+\mathrm{i} \lambda)) \gtrsim \lambda^{2}\left(\sigma^{2}+\int_{(0,1 / \lambda)} s^{2} \mathrm{e}^{-w s} \nu(\mathrm{d} s)\right)
$$

By Lemma 1 we obtain, for $\lambda \geqslant w$,

$$
\operatorname{Re}(\varphi(w)-\varphi(w+\mathrm{i} \lambda)) \gtrsim \lambda^{2}\left(\sigma^{2}+\int_{(0,1 / \lambda)} s^{2} \nu(\mathrm{d} s)\right) \gtrsim \lambda^{2} \varphi^{\prime \prime}(\lambda) .
$$

If $w>\lambda>0$, then by (2.16)

$$
\operatorname{Re}(\varphi(w)-\varphi(w+\mathrm{i} \lambda)) \gtrsim \lambda^{2}\left(\sigma^{2}+\int_{(0,1 / w)} s^{2} \mathrm{e}^{-w s} \nu(\mathrm{d} s)\right) \geqslant \mathrm{e}^{-1} \lambda^{2}\left(\sigma^{2}+\int_{(0,1 / w)} s^{2} \nu(\mathrm{d} s)\right)
$$

which, together with Lemma 1, ends the proof.

\section{Asymptotics}

Theorem 1. Let $\mathbf{X}$ be a spectrally positive Lévy processes of unbounded variation with Laplace exponent $\varphi$. Suppose that $\varphi^{\prime \prime} \in \mathrm{WLSC}\left(\alpha-2, c, x_{0}\right)$ for some $c \in(0,1], x_{0} \geqslant 0$, and $\alpha>0$. Then the probability distribution of $X_{t}$ is absolutely continuous for all $t>0$. If we denote its density by $p(t, \cdot)$, then for each $\varepsilon>0$, 
there is $M_{0}>0$ such that

$$
\left|p\left(t,-t \varphi^{\prime}(w)\right) \sqrt{2 \pi t \varphi^{\prime \prime}(w)} \exp \left\{t\left(w \varphi^{\prime}(w)-\varphi(w)\right)\right\}-1\right| \leqslant \varepsilon,
$$

provided that $w>x_{0}$ and $t w^{2} \varphi^{\prime \prime}(w)>M_{0}$.

Remark 1. Suppose $\sigma=0$. It is known (see, e.g., [8, Lemma 2.9]) that the scaling property with the scaling index $\alpha \geqslant 1$ implies unbounded variation. However, Theorem 1 holds in greater generality. Namely, there are processes of unbounded variation that satisfy the scaling condition with $\alpha$ strictly smaller than 1 , and we may construct a Lévy measure that satisfies (2.4) and whose corresponding second derivative of the Laplace exponent has lower and upper Matuszewska indices of orders $-3 / 2$ and $-1 / 2$, respectively. Thus the lower scaling condition for $\varphi^{\prime \prime}$ holds only for $\alpha<1$. An example of such a process is constructed in Example 2. We also note that the Gaussian component is not excluded.

Proof of Theorem 1. Let $x=-t \varphi^{\prime}(w)$ and $M>0$. We first show that

$$
p(t, x)=\frac{1}{2 \pi} \cdot \frac{\mathrm{e}^{-\Theta(x / t, 0)}}{\sqrt{t \varphi^{\prime \prime}(w)}} \int_{\mathbb{R}} \exp \left\{-t\left(\Theta\left(\frac{x}{t}, \frac{u}{\sqrt{t \varphi^{\prime \prime}(w)}}\right)-\Theta\left(\frac{x}{t}, 0\right)\right)\right\} \mathrm{d} u,
$$

provided that $w>x_{0}$ and $t w^{2} \varphi^{\prime \prime}(w)>M$, where for $\lambda>0$, we have set

$$
\Theta\left(\frac{x}{t}, \lambda\right)=-\left(\varphi(w+\mathrm{i} \lambda)+\frac{x}{t}(w+\mathrm{i} \lambda)\right) .
$$

To this end, we recall that, by the Mellin inversion formula, if the limit

$$
\lim _{L \rightarrow \infty} \frac{1}{2 \pi \mathrm{i}} \int_{w-i L}^{w+i L} \mathrm{e}^{t \varphi(\lambda)+\lambda x} \mathrm{~d} \lambda \text { exists }
$$

then the probability distribution of $X_{t}$ has a density $p(t, \cdot)$, and

$$
p(t, x)=\lim _{L \rightarrow \infty} \frac{1}{2 \pi \mathrm{i}} \int_{w-i L}^{w+i L} \mathrm{e}^{t \varphi(\lambda)+\lambda x} \mathrm{~d} \lambda .
$$

Using change of variables twice, we obtain

$$
\begin{aligned}
\frac{1}{2 \pi \mathrm{i}} \int_{w-\mathrm{i} L}^{w+i L} \mathrm{e}^{t \varphi(\lambda)+\lambda x} \mathrm{~d} \lambda & =\frac{1}{2 \pi} \int_{-L}^{L} \mathrm{e}^{-t \Theta(x / t, \lambda)} \mathrm{d} \lambda \\
& =\frac{\mathrm{e}^{-t \Theta(x / t, 0)}}{2 \pi} \int_{-L}^{L} \exp \left\{-t\left(\Theta\left(\frac{x}{t}, \lambda\right)-\Theta\left(\frac{x}{t}, 0\right)\right)\right\} \mathrm{d} \lambda \\
& =\frac{\mathrm{e}^{-t \Theta(x / t, 0)}}{2 \pi \sqrt{t \varphi^{\prime \prime}(w)}} \int_{-L \sqrt{t \varphi^{\prime \prime}(w)}}^{L \sqrt{t \varphi^{\prime \prime}(w)}} \exp \left\{-t\left(\Theta\left(\frac{x}{t}, \frac{u}{\sqrt{t \varphi^{\prime \prime}(w)}}\right)-\Theta\left(\frac{x}{t}, 0\right)\right)\right\} \mathrm{d} u
\end{aligned}
$$


Next, we note that there is $C>0$, not depending on $M$, such that for all $u \in \mathbb{R}$,

$$
t \operatorname{Re}\left(\Theta\left(\frac{x}{t}, \frac{u}{\sqrt{t \varphi^{\prime \prime}(w)}}\right)-\Theta\left(\frac{x}{t}, 0\right)\right) \geqslant C\left(u^{2} \wedge\left(|u|^{\alpha} M^{1-\alpha / 2}\right)\right)
$$

provided that $w>x_{0}$ and $t w^{2} \varphi^{\prime \prime}(w)>M$. Indeed, by (3.2) and Lemma 2, for $w>x_{0}$, we get

$$
t \operatorname{Re}\left(\Theta\left(\frac{x}{t}, \frac{u}{\sqrt{t \varphi^{\prime \prime}(w)}}\right)-\Theta\left(\frac{x}{t}, 0\right)\right) \gtrsim \frac{|u|^{2}}{\varphi^{\prime \prime}(w)} \varphi^{\prime \prime}\left(\frac{|u|}{\sqrt{t \varphi^{\prime \prime}(w)}} \vee w\right)
$$

and (3.4) follows by the scaling property of $\varphi^{\prime \prime}$. Hence (3.3) follows from the dominated convergence theorem. Consequently, Mellin's inversion formula yields (3.1).

Next, we prove that for each $\varepsilon>0$, there is $M_{0}>0$ such that

$$
\left|\int_{\mathbb{R}} \exp \left\{-t\left(\Theta\left(\frac{x}{t}, \frac{u}{\sqrt{t \varphi^{\prime \prime}(w)}}\right)-\Theta\left(\frac{x}{t}, 0\right)\right)\right\} \mathrm{d} u-\int_{\mathbb{R}} \mathrm{e}^{-u^{2} / 2} \mathrm{~d} u\right| \leqslant \varepsilon,
$$

provided that $w>x_{0}$ and $t w^{2} \varphi^{\prime \prime}(w)>M_{0}$. In view of (3.4), taking $M_{0}>1$ sufficiently large, we get

$$
\left|\int_{|u| \geqslant M_{0}^{1 / 4}} \exp \left\{-t\left(\Theta\left(\frac{x}{t}, \frac{u}{\sqrt{t \varphi^{\prime \prime}(w)}}\right)-\Theta\left(\frac{x}{t}, 0\right)\right)\right\} \mathrm{d} u\right| \leqslant \int_{|u| \geqslant M_{0}^{1 / 4}} \mathrm{e}^{-C|u|^{\alpha}} \mathrm{d} u \leqslant \varepsilon
$$

and

$$
\int_{|u| \geqslant M_{0}^{1 / 4}} \mathrm{e}^{-u^{2} / 2} \mathrm{~d} u \leqslant \varepsilon
$$

Next, note that there is $C>0$ such that

$$
\left.\left.\left|t\left(\Theta\left(\frac{x}{t}, \frac{u}{\sqrt{t \varphi^{\prime \prime}(w)}}\right)-\Theta\left(\frac{x}{t}, 0\right)\right)-\frac{1}{2}\right| u\right|^{2}|\leqslant C| u\right|^{3} M_{0}^{-1 / 2} .
$$

Indeed, since $\partial_{\lambda} \Theta(x / t, 0)=0$, by Taylor's formula we get

$$
\begin{aligned}
\mid t & \left(\Theta\left(\frac{x}{t}, \frac{u}{\sqrt{t \varphi^{\prime \prime}(w)}}\right)-\Theta\left(\frac{x}{t}, 0\right)\right)-\frac{1}{2}|u|^{2} \mid \\
& =\left.\left|\frac{1}{2} \partial_{\lambda}^{2} \Theta\left(\frac{x}{t}, \xi\right) \frac{|u|^{2}}{\varphi^{\prime \prime}(w)}-\frac{1}{2}\right| u\right|^{2}\left|=\frac{|u|^{2}}{2 \varphi^{\prime \prime}(w)}\right| \varphi^{\prime \prime}(w)-\varphi^{\prime \prime}(w+\mathrm{i} \xi) \mid,
\end{aligned}
$$

where $\xi$ is a number satisfying $|\xi| \leqslant|u| / \sqrt{t \varphi^{\prime \prime}(w)}$. We also have

$$
\begin{aligned}
\left|\varphi^{\prime \prime}(w)-\varphi^{\prime \prime}(w+\mathrm{i} \xi)\right| & \leqslant \int_{(0, \infty)} s^{2} \mathrm{e}^{-w s}\left|\mathrm{e}^{-\mathrm{i} \xi s}-1\right| \nu(\mathrm{d} s) \leqslant 2|\xi| \int_{(0, \infty)} s^{3} \mathrm{e}^{-w s} \nu(\mathrm{d} s) \\
& =2|\xi|\left(-\varphi^{\prime \prime \prime}(w)\right) .
\end{aligned}
$$


Since $\varphi^{\prime \prime}$ is doubling, by [7, Prop. 2.1], for $w>x_{0}, \varphi^{\prime \prime}(w) \gtrsim w\left(-\varphi^{\prime \prime \prime}(w)\right)$, which, together with the estimate on $|\xi|$, yields

$$
\left|\varphi^{\prime \prime}(w)-\varphi^{\prime \prime}(w+\mathrm{i} \xi)\right| \leqslant C \frac{|u|}{\sqrt{t \varphi^{\prime \prime}(w)}} \cdot \frac{\varphi^{\prime \prime}(w)}{w} \leqslant C M_{0}^{-1 / 2}|u| \varphi^{\prime \prime}(w)
$$

if only $t w^{2} \varphi^{\prime \prime}(w)>M_{0}$, proving (3.8) through (3.9). Finally, since for any $z \in \mathbb{C},\left|\mathrm{e}^{z}-1\right| \leqslant|z| \mathrm{e}^{|z|}$, (3.8) implies

$$
\begin{aligned}
& \left|\int_{|u|<M_{0}^{1 / 4}} \exp \left\{-t\left(\Theta\left(\frac{x}{t}, \frac{u}{\sqrt{t \varphi^{\prime \prime}(w)}}\right)-\Theta\left(\frac{x}{t}, 0\right)\right)\right\} \mathrm{d} u-\int_{|u|<M_{0}^{1 / 4}} \mathrm{e}^{-u^{2} / 2} \mathrm{~d} u\right| \\
& \leqslant C M_{0}^{-1 / 2} \int_{|u|<M_{0}^{1 / 4}} \exp \left\{-\frac{1}{2}|u|^{2}+C M_{0}^{-1 / 2}|u|^{3}\right\}|u|^{3} \mathrm{~d} u \leqslant \varepsilon,
\end{aligned}
$$

provided that $M_{0}$ is sufficiently large, which, together with (3.6) and (3.7), completes the proof of (3.5), and the theorem follows.

Remark 2. If $x_{0}=0$, then the constant $M_{0}$ in Theorem 1 depends only on $\alpha$ and $c$. If $x_{0}>0$, then it also depends on

$$
\sup _{x \in\left[x_{0}, 2 x_{0}\right]} \frac{x\left(-\varphi^{\prime \prime \prime}(x)\right)}{\varphi^{\prime \prime}(x)} .
$$

Corollary 4. Suppose that $\varphi^{\prime \prime} \in \operatorname{WLSC}\left(\alpha-2, c, x_{0}\right)$ for some $c \in(0,1], x_{0} \geqslant 0$, and $\alpha>0$. Then there is $M_{0}>0$ such that

$$
p(t, x) \approx \frac{1}{\sqrt{t \varphi^{\prime \prime}(w)}} \exp \left\{-t\left(w \varphi^{\prime}(w)-\varphi(w)\right)\right\}
$$

uniformly on the set

$$
\left\{(t, x) \in \mathbb{R}_{+} \times \mathbb{R}: x<-t \varphi^{\prime}\left(x_{0}\right) \text { and } t w^{2} \varphi^{\prime \prime}(w)>M_{0}\right\},
$$

where $w=\left(\varphi^{\prime}\right)^{-1}(-x / t)$.

Corollary 5. Suppose that $\varphi^{\prime \prime} \in \operatorname{WLSC}\left(\alpha-2, c, x_{0}\right)$ for some $c \in(0,1], x_{0} \geqslant 0$, and $\alpha>1$. Assume also that $\varphi^{\prime}\left(\theta_{1}\right)=0$. Then there is $M>0$ such that

$$
p(t, x) \approx \frac{1}{\sqrt{t \varphi^{\prime \prime}(w)}} \exp \left\{-t\left(w \varphi^{\prime}(w)-\varphi(w)\right)\right\}
$$

uniformly on the set

$$
\left\{(t, x) \in \mathbb{R}_{+} \times \mathbb{R}:-x \varphi^{-1}\left(\frac{1}{t}\right)>M \text { and } 0 \leqslant t \varphi\left(x_{0} \vee 2 \theta_{0}\right) \leqslant 1\right\},
$$

where $w=\left(\varphi^{\prime}\right)^{-1}(-x / t)$.

Remark 3. The condition $\varphi^{\prime}\left(\theta_{1}\right)=0$ covers the case $\mathbf{E} X_{1} \in[0, \infty]$. For the case $\mathbf{E} X_{1}>0$, it is not, however, optimal, because we do not treat positive $x$ that may be in the area from Corollary 4 . We also note that $\alpha=2$ is included. 
Proof. We verify that for $(t, x)$ belonging to the set (3.10), we have $w>x_{0}$ and $w t \varphi^{\prime \prime}(w)>M_{0}$, where $w=\left(\varphi^{\prime}\right)^{-1}(-x / t)$. Let $M \geqslant C_{1} C_{2}$, where $C_{1}$ and $C_{2}$ are taken from Propositions 2 and 1, respectively. By Propositions 2 and 1 we have

$$
\begin{aligned}
\frac{-x}{t} & >M \frac{1}{t \varphi^{-1}(1 / t)}=M \frac{\varphi\left(\varphi^{-1}(1 / t)\right)}{\varphi^{-1}(1 / t)} \geqslant C_{1}\left(C_{1}^{-1} C_{2}^{-1} M\right) \varphi^{\prime}\left(\varphi^{-1}\left(\frac{1}{t}\right)\right) \\
& \geqslant \varphi^{\prime}\left(C_{1}^{-1} C_{2}^{-1} M \varphi^{-1}\left(\frac{1}{t}\right)\right) .
\end{aligned}
$$

Thus

$$
w=\left(\varphi^{\prime}\right)^{-1}\left(-\frac{x}{t}\right)>C_{1}^{-1} C_{2}^{-1} M \varphi^{-1}\left(\frac{1}{t}\right)
$$

In particular, $w>x_{0} \vee 2 \theta_{0}$. Next, by Corollary 2 there is $c_{1} \in(0,1]$ such that $t w^{2} \varphi^{\prime \prime}(w) \geqslant c_{1} t \varphi(w)$. Since, by Proposition 3 , there is $c_{2} \in(0,1]$ such that $\varphi \in \operatorname{WLSC}\left(\alpha, c_{2}, x_{0} \vee \theta_{0}\right)$, we obtain

$$
t \varphi(w) \geqslant c_{2}\left(\frac{w}{\varphi^{-1}(1 / t)}\right)^{\alpha}
$$

In view of (3.11), we get that

$$
t w^{2} \varphi^{\prime \prime}(w)>c_{1} c_{2}\left(C_{1}^{-1} C_{2}^{-1} M\right)^{\alpha}>M_{0},
$$

provided that $M$ is sufficiently large. Applying Theorem 1 yields the desired result.

\section{Upper and lower estimates on the density}

In this section, we assume that $\varphi^{\prime \prime} \in \operatorname{WLSC}\left(\alpha-2, c, x_{0}\right)$ for some $c \in(0,1], x_{0} \geqslant 0$, and $\alpha>0$. By Theorem 1 the probability distribution $X_{t}$ has a density $p(t, \cdot)$. Let us define $\Phi(x)=x^{2} \varphi^{\prime \prime}(x), x>0$. Clearly, $\Phi \in \operatorname{WLSC}\left(\alpha, c, x_{0}\right)$. By $\Phi^{-1}$ we denote its right-sided inverse, that is,

$$
\Phi^{-1}(s)=\sup \left\{r>0: \Phi^{*}(r)=s\right\},
$$

where $\Phi^{*}(r)=\sup _{0<s \leqslant r} \Phi(s)$. Clearly, $\Phi^{-1}$ is nondecreasing. Similarly to $\psi^{-1}$, we have

$$
\Phi^{*}\left(\Phi^{-1}(s)\right)=s, \quad \Phi^{-1}\left(\Phi^{*}(s)\right) \geqslant s .
$$

Note that since for all $x>0$ and $\lambda \geqslant 1$,

$$
\Phi(\lambda x) \leqslant \lambda^{2} \Phi(x)
$$

we obtain

$$
\Phi^{*}(\lambda x) \leqslant \lambda^{2} \Phi^{*}(x) .
$$

Furthermore, for any $r>0$, let $u$ be such that $\Phi^{-1}(r)=u$. Then by (4.2) and (4.1), for any $\lambda \geqslant 1$,

$$
\Phi^{-1}(\lambda r)=\Phi^{-1}\left(\lambda \Phi^{*}(u)\right) \geqslant \Phi^{-1}\left(\Phi^{*}(\sqrt{\lambda} u)\right) \geqslant \sqrt{\lambda} u .
$$

Thus, for any $r>0$ and $\lambda \geqslant 1$,

$$
\Phi^{-1}(\lambda r) \geqslant \sqrt{\lambda} \Phi^{-1}(r) .
$$

Let us start with a following observation on the comparability of concentration functions $K$ and $h$. 
Proposition 5. Suppose $\varphi^{\prime \prime} \in \operatorname{WLSC}\left(\alpha-2, c, x_{0}\right)$ for some $c \in(0,1], x_{0} \geqslant 0$, and $\alpha>0$. Then there is $C \geqslant 1$ such that for all $0<r<1 / x_{0}$,

$$
K(r) \leqslant h(r) \leqslant C K(r)
$$

Proof. Since $K(r) \leqslant h(r)$, it suffices to show that there is $C \geqslant 1$ such that for all $0<r<1 / x_{0}$,

$$
h(r) \leqslant C K(r) .
$$

In view of (2.7), we have

$$
h(r)=2 \int_{r}^{1 / x_{0}} K(s) \frac{\mathrm{d} s}{s}+2 \int_{1 / x_{0}}^{\infty} K(s) \frac{\mathrm{d} s}{s} .
$$

By Corollary 3 we have $K(r) \approx \Phi(1 / r)$ for $0<r<1 / x_{0}$, which, together with the scaling property, implies

$$
\int_{r}^{1 / x_{0}} K(s) \frac{\mathrm{d} s}{s} \lesssim K(r), \quad 0<r<\frac{1}{x_{0}} .
$$

That finishes the proof for the case $x_{0}=0$. If $x_{0}>0$, then we observe that we also have $K(r) \gtrsim 1$ for all $0<r<1 / x_{0}$. Since the second term on the right-hand side of (4.4) is constant, the proof is finished.

In view of (2.8), Proposition 5, and Corollary 3, we have

$$
\psi^{*}(x) \approx h\left(\frac{1}{x}\right) \approx K\left(\frac{1}{x}\right) \approx \Phi(x)
$$

for all $x>x_{0}$. In particular, $\psi^{*} \in \operatorname{WLSC}\left(\alpha, c, x_{0}\right)$ for some $c \in(0,1]$. Furthermore, for all $x>x_{0}$,

$$
\psi^{*}(x) \lesssim K\left(\frac{1}{x}\right)=x^{2} \int_{(0,1 / x)} s^{2} \nu(\mathrm{d} s) \lesssim \int_{(0,1 / x)}(1-\cos s x) \nu(\mathrm{d} s) .
$$

Thus, for all $x>x_{0}$,

$$
\psi^{*}(x) \lesssim \operatorname{Re} \psi(x)
$$

As a corollary, we now present the aforementioned equivalence between the scaling property of the second derivative of the Laplace exponent and the real part of the characteristic exponent.

Corollary 6. We have $\varphi^{\prime \prime} \in \operatorname{WLSC}\left(\alpha-2, c, x_{0}\right)$ for some $c \in(0,1], x_{0} \geqslant 0$, and $\alpha>0$ if and only if $\operatorname{Re} \psi \in \mathrm{WLSC}\left(\alpha, \tilde{c}, x_{0}\right)$ for some $\tilde{c} \in(0,1]$.

Proof. In view of (4.5) and (4.6), it remains to prove the second implication in the corollary. We first prove that $\psi^{*} \in \operatorname{WLSC}\left(\alpha, c_{1}, x_{0}\right)$ for some $c_{1} \in(0,1]$. Let $x \geqslant x_{0}$ and $\lambda \geqslant 1$. By the scaling property of $\operatorname{Re} \psi$ we have

$$
\psi^{*}(\lambda x)=\max \left\{\psi^{*}\left(\lambda x_{0}\right), \sup _{\lambda x_{0}<x \leqslant \lambda x} \operatorname{Re} \psi(r)\right\} \gtrsim \max \left\{\psi^{*}\left(x_{0}\right), \lambda^{\alpha} \sup _{x_{0}<r \leqslant x} \operatorname{Re} \psi(r)\right\}
$$


Now note that since $\lim _{r \rightarrow \infty} \operatorname{Re} \psi(r)=\infty$, there is $x_{1} \geqslant x_{0}$ such that $\operatorname{Re} \psi(x) \geqslant \psi^{*}\left(x_{0}\right)$ for all $x \geqslant x_{1}$, and, consequently, for all $\lambda \geqslant 1$ and $x \geqslant x_{1}$,

$$
\psi^{*}(\lambda x) \gtrsim \max \left\{\psi^{*}\left(x_{0}\right), \lambda^{\alpha} \sup _{r \leqslant x} \operatorname{Re} \psi(r)\right\}=\lambda^{\alpha} \psi^{*}(x),
$$

and by standard extension argument we get the scaling property of $\psi^{*}$, as desired.

It remains to notice that, by the integral representation of $\varphi^{\prime \prime}$,

$$
x^{-2} K\left(\frac{1}{x}\right) \lesssim \varphi^{\prime \prime}(x) \lesssim x^{-2} h\left(\frac{1}{x}\right)
$$

Thus [8, Lemma 2.3] yields the claim.

Proposition 6. Suppose $\varphi^{\prime \prime} \in \operatorname{WLSC}\left(\alpha-2, c, x_{0}\right)$ for some $c \in(0,1], x_{0} \geqslant 0$, and $\alpha>0$. Then for all $r>2 h\left(1 / x_{0}\right)$,

$$
\frac{1}{h^{-1}(r)} \approx \psi^{-1}(r)
$$

Furthermore, there is $C \geqslant 1$ such that for all $\lambda \geqslant 1$ and $r>2 h\left(1 / x_{0}\right)$,

$$
\psi^{-1}(\lambda r) \leqslant C \lambda^{1 / \alpha} \psi^{-1}(r)
$$

Proof. Follows immediately by (2.8) and the scaling property of $h^{-1}$ provided by Proposition 5 and [8, Lemma 2.3].

Proposition 7. Suppose $\varphi^{\prime \prime} \in \operatorname{WLSC}\left(\alpha-2, c, x_{0}\right)$ for some $c \in(0,1], x_{0} \geqslant 0$, and $\alpha>0$. Then for all $x>x_{0}$,

$$
\psi^{*}(x) \approx \Phi^{*}(x)
$$

and for all $r>\Phi\left(x_{0}\right)$,

$$
\psi^{-1}(r) \approx \Phi^{-1}(r) .
$$

Furthermore, there is $C \geqslant 1$ such that for all $\lambda \geqslant 1$ and $r>\Phi\left(x_{0}\right)$,

$$
\Phi^{-1}(\lambda r) \leqslant C \lambda^{1 / \alpha} \Phi^{-1}(r) .
$$

Proof. The first inequality of (4.7) follows immediately from (4.5). If $x_{0}=0$, then the second inequality is also a consequence of (4.5). If this is not the case, then note that for $x>x_{0}$,

$$
\begin{aligned}
\Phi^{*}(x) & =\max \left\{\sup _{0<y \leqslant x_{0}} \Phi(y), \sup _{x_{0}<y \leqslant x} \Phi(y)\right\} \lesssim \max \left\{\Phi^{*}\left(x_{0}\right), \psi^{*}(x)\right\} \\
& \leqslant\left(1+\frac{\Phi^{*}\left(x_{0}\right)}{\psi^{*}\left(x_{0}\right)}\right) \psi^{*}(x),
\end{aligned}
$$

and the first part is proved. Furthermore, it follows that $\psi^{-1}\left(C^{-1} r\right) \leqslant \Phi^{-1}(r) \leqslant \psi^{-1}(C r)$ for all $r>$ $C \psi^{*}\left(x_{0}\right)$. Hence, by Proposition $6, \Phi^{-1}(r) \approx \psi^{-1}(r)$ for all $r>C \max \left\{\psi^{*}\left(x_{0}\right), 2 h\left(1 / x_{0}\right)\right\}$, and (4.8) follows by standard extension argument. The scaling property of $\Phi^{-1}$ is an easy consequence of (4.8) and Proposition 6.

Since $\Phi \leqslant \Phi^{*}$, by Proposition 7 and (4.5) we immediately obtain the following: 
Remark 4. Suppose $\varphi^{\prime \prime} \in \operatorname{WLSC}\left(\alpha-2, c, x_{0}\right)$ for some $c \in(0,1], x_{0} \geqslant 0$, and $\alpha>0$. There is $c_{1} \in(0,1]$ such that for all $x>x_{0}$,

$$
c_{1} \Phi^{*}(x) \leqslant \Phi(x) \leqslant \Phi^{*}(x) .
$$

Proposition 8. Suppose that $\varphi^{\prime \prime} \in \operatorname{WLSC}\left(\alpha-2, c, x_{0}\right)$ for some $c \in(0,1], x_{0} \geqslant 0$, and $\alpha>1$. Assume also that $\varphi^{\prime}\left(\theta_{1}\right)=0$. Then for all $x>x_{0} \vee 2 \theta_{0}$,

$$
\Phi^{*}(x) \approx \varphi(x),
$$

and for all $r>\Phi\left(x_{0} \vee 2 \theta_{0}\right)$,

$$
\Phi^{-1}(r) \approx \varphi^{-1}(r)
$$

Proof. Corollary 2 and Remark 4 yield the first part. The proof of the second is omitted due to similarity to the proof of Proposition 7.

\subsection{Upper estimates}

From this moment on, we additionally assume that $\sigma=0$. As explained in Preliminaries, that is equivalent to saying that $\mathbf{X}$ satisfies the integral condition (1.1). Suppose $\varphi^{\prime \prime} \in \operatorname{WLSC}\left(\alpha-2, c, x_{0}\right)$. Recall that since $\varphi^{\prime \prime}$ is positive and continuous on $(0, \infty)$, if $x_{0}>0$, then at the cost of worsening the constant $c$, we can extend the area of comparability to any $x_{1} \in\left(0, x_{0}\right)$ so that $\varphi^{\prime \prime} \in \mathrm{WLSC}\left(\alpha-2, c^{\prime}, x_{1}\right)$, where $c^{\prime}$ depends on $x_{1}$. Thus if $\theta_{1}>0$ and $x_{0}>0$, then we may and do assume that $x_{0}$ is shifted so that $x_{0} \leqslant \theta_{1}$. With this in mind, let us define $\eta:[0, \infty) \mapsto[0, \infty]$ as

$$
\eta(s)= \begin{cases}\infty & \text { if } s=0 \\ s^{-1} \Phi^{*}\left(\frac{1}{s}\right) & \text { if } 0<s<x_{0}^{-1} \\ A s^{-1}\left|\varphi\left(\frac{1}{s}\right)\right| & \text { if } x_{0}^{-1} \leqslant s\end{cases}
$$

where $A=\Phi^{*}\left(x_{0}\right) /\left|\varphi\left(x_{0}\right)\right|$.

Let us comment on the function $\eta$. As we will see in Theorem 2, it will play a role of majorant on the transition density. In such a setting, it is clear that $\eta$ must be nonnegative. Moreover, in the proof, we will need it to be monotone. Now, if $\theta_{1}>0$, then we know that $\varphi$ is indeed monotone for $x \in\left(0, \theta_{1}\right)$ but also negative. Thus a change of sign is required. On the other hand, if $\theta_{1}=0$, then $\varphi \geqslant 0$, and there is no need for absolute value and shifting of $x_{0}$. In general, however, $\varphi$ may be negative in a neighborhood of the origin and change sign at $\theta_{0}$, so we have to be careful in expanding the scaling area to the proper place. Note that by Corollary 1 and Remark 4, $A \leqslant c^{\prime}$, where $c^{\prime}$ depends only on $\theta_{1}$.

Denote

$$
b_{r}=b+\int_{(0, \infty)} s\left(\mathbf{1}_{s<r}-\mathbf{1}_{s<1}\right) \nu(\mathrm{d} s) .
$$

Theorem 2. Let $\mathbf{X}$ be a spectrally positive Lévy process of infinite variation with Lévy-Khintchine exponent $\psi$ and Laplace exponent $\varphi$. Suppose that $\sigma=0$ and $\varphi^{\prime \prime} \in \operatorname{WLSC}\left(\alpha-2, c, x_{0}\right)$ for some $c \in(0,1], x_{0} \geqslant 0$, and $\alpha>0$. We also assume that $\nu(d x)$ has an almost monotone density $\nu(x)$. Then the probability distribution of $X_{t}$ has a density $p(t, \cdot)$. Moreover, there is $C>0$ such that for all $t \in\left(0,1 / \Phi\left(x_{0}\right)\right)$ and $x \in \mathbb{R}$,

$$
p\left(t, x+t b_{1 / \psi^{-1}(1 / t)}\right) \leqslant C \min \left\{\Phi^{-1}\left(\frac{1}{t}\right), t \eta(|x|)\right\} .
$$


Proof. In the first step, we verify the assumptions of [9, Thm. 2.1]. First, note that for any $\lambda>0$,

$$
\varphi^{\prime \prime}(\lambda) \geqslant \int_{0}^{1 / \lambda} s^{2} \mathrm{e}^{-\lambda s} \nu(s) \mathrm{d} s \gtrsim \nu\left(\frac{1}{\lambda}\right) \lambda^{-3},
$$

and thus, by Corollary $1, \nu(x) \lesssim \eta(x)$ for all $x>0$. Since $\eta$ is nonincreasing, we conclude that the first assumption is satisfied. Next, we claim that $\eta$ has a doubling property on $(0, \infty)$. Indeed, since $\varphi^{\prime \prime}$ is nonincreasing, by Remark 4, for $0<s<x_{0}^{-1}$, we have

$$
\eta\left(\frac{1}{2} s\right) \approx 4 s^{-3} \varphi^{\prime \prime}\left(\frac{2}{s}\right) \lesssim s^{-3} \varphi^{\prime \prime}\left(\frac{1}{s}\right) \approx \eta(s) .
$$

This completes the argument for the case $x_{0}=0$. If $x_{0}>0$, then Proposition 1 (or (2.10) if $\theta_{0}>0$ ) yields the claim for $s>2 x_{0}^{-1}$. Lastly, the function $\left[x_{0} / 2, x_{0}\right] \ni x \mapsto \Phi^{*}(2 x) /|\varphi(x)|$ is continuous and hence bounded.

Therefore, since $s \wedge|x|-|x| / 2 \geqslant s / 2$ for $s>0$ and $x \in \mathbb{R}$, the doubling property of $\eta$ and (4.5) imply the second assumption. Finally, since $\psi^{*}$ has the weak lower scaling property and satisfies (4.6), by [8, Thm. 3.1] and Proposition 6 there are $C>0$ and $t_{1} \in(0, \infty]$ such that for all $t \in\left(0, t_{1}\right)$,

$$
\int_{\mathbb{R}} \mathrm{e}^{-t \operatorname{Re} \psi(\xi)} \mathrm{d} \xi \leqslant C \psi^{-1}\left(\frac{1}{t}\right)
$$

with $t_{1}=\infty$ whenever $x_{0}=0$. Note that if $t_{1}<48 / \Phi\left(x_{0}\right)$, then using the positivity and monotonicity, we can expand the estimate for $t_{1} \leqslant t<48 / \Phi\left(x_{0}\right)$, and the first step is finished.

Therefore by $\left[9\right.$, Thm. 2.1] there is $C>0$ such that for all $t \in\left(0,1 / \Phi\left(x_{0}\right)\right)$ and $x \in \mathbb{R}$,

$$
p\left(t, x+t b_{1 / \psi^{-1}(1 / t)}\right) \leqslant C \psi^{-1}\left(\frac{1}{t}\right) \min \left\{1, t\left(\psi^{-1}\left(\frac{1}{t}\right)\right)^{-1} \eta(|x|)+\left(1+|x| \psi^{-1}\left(\frac{1}{t}\right)\right)^{-3}\right\} .
$$

Now it suffices to prove that

$$
\frac{\psi^{-1}(1 / t)}{\left(1+|x| \psi^{-1}(1 / t)\right)^{3}} \lesssim t \eta(|x|)
$$

whenever $\operatorname{t\eta }(|x|) \leqslant\left(A / c^{\prime}\right) \Phi^{-1}(1 / t)$.

First, note that for any $\varepsilon \in(0,1]$, the condition $t \eta(|x|) \leqslant\left(A \varepsilon / c^{\prime}\right) \Phi^{-1}(1 / t)$ implies

$$
t \Phi^{*}\left(\frac{1}{|x|}\right) \leqslant \varepsilon|x| \Phi^{-1}\left(\frac{1}{t}\right) .
$$

Indeed, by Corollary 1 and Remark 4 we have $|x| \eta(|x|) \geqslant \frac{A}{c^{\prime}} \Phi^{*}(1 /|x|)$, which entails (4.12). Furthermore, we have $\varepsilon^{1 / 3}|x| \Phi^{-1}(1 / t) \geqslant 1$, since otherwise by (4.2) we would have

$$
1<t \Phi^{*}\left(\frac{1}{\varepsilon^{1 / 3}|x|}\right) \leqslant \frac{1}{\varepsilon^{2 / 3}} t \Phi^{*}\left(\frac{1}{|x|}\right),
$$

which in turn would yield

$$
\varepsilon^{1 / 3}|x| \Phi^{-1}\left(\frac{1}{t}\right)<\varepsilon^{-2 / 3} t \Phi^{*}\left(\frac{1}{|x|}\right)
$$

contrary to (4.12). 
Now suppose $t \eta(|x|) \leqslant\left(A / c^{\prime}\right) \Phi^{-1}(1 / t)$. Since $|x| \Phi^{-1}(1 / t) \geqslant 1$, by (4.2) we infer that

$$
t \Phi^{*}(1 /|x|)=\frac{\Phi^{*}(1 /|x|)}{\Phi^{*}\left(|x| \Phi^{-1}(1 / t) \cdot 1 /|x|\right)} \geqslant \frac{1}{\left(|x| \Phi^{-1}(1 / t)\right)^{2}} \geqslant \frac{|x| \Phi^{-1}(1 / t)}{\left(1+|x| \Phi^{-1}(1 / t)\right)^{3}} .
$$

It remains to notice that Proposition 7 entails (4.11), and the proof is completed.

Remark 5. In Theorem 2, we may replace $b_{1 / \psi^{-1}(1 / t)}$ by $b_{1 / \Phi^{-1}(1 / t)}$. Indeed, if $0<r_{1} \leqslant r_{2}<1 / \Phi\left(x_{0}\right)$, then by (4.5) and Proposition 7 we have

$$
\left|b_{r_{1}}-b_{r_{2}}\right| \leqslant \int_{\left(r_{1}, r_{2}\right]} s \nu(\mathrm{d} s) \leqslant r_{1}^{-1} r_{2}^{2} h\left(r_{2}\right) \lesssim r_{1}^{-1} r_{2}^{2} \psi^{*}\left(r_{2}^{-1}\right) \lesssim r_{1}^{-1} r_{2}^{2} \Phi^{*}\left(r_{2}^{-1}\right) .
$$

Thus, again by Proposition 7, there is $C \geqslant 1$ such that for all $t \in\left(0,1 / \Phi\left(x_{0}\right)\right)$,

$$
\left|b_{1 / \psi^{-1}(1 / t)}-b_{1 / \Phi^{-1}(1 / t)}\right| \leqslant \frac{C}{t \Phi^{-1}(1 / t)} .
$$

Now recall that if $\operatorname{t\eta }(|x|) \leqslant\left(A \varepsilon / c^{\prime}\right) \Phi^{-1}(1 / t)$, then by the proof of Theorem 2 we have $|x| \Phi^{-1}(1 / t) \geqslant \varepsilon^{-1 / 3}$. Therefore by taking $\varepsilon=(2 C)^{-3}$ we obtain $|x| \geqslant 2 C / \Phi^{-1}(1 / t)$, and by the monotonicity and doubling property of $\eta$ we conclude that

$$
\eta\left(\left|x+t\left(b_{1 / \psi^{-1}(1 / t)}-b_{1 / \Phi^{-1}(1 / t)}\right)\right|\right) \lesssim \eta(|x|) .
$$

\subsection{Lower estimates}

We begin with an estimate, which, together with Theorem 2, will allow us to localize the supremum of $p(t, \cdot)$. Note that here we require the scaling condition with $\alpha \geqslant 1$.

Lemma 3. Let $\mathbf{X}$ be a spectrally positive Lévy process of infinite variation with Laplace exponent $\varphi$. Suppose that $\sigma=0$ and $\varphi^{\prime \prime} \in \operatorname{WLSC}\left(\alpha-2, c, x_{0}\right)$ for some $c \in(0,1], x_{0} \geqslant 0$, and $\alpha \geqslant 1$. Then there is $M_{0}>1$ such that for all $M \geqslant M_{0}$ and $\rho_{1}, \rho_{2}>0$, there exists $C>0$ such that for all $t \in\left(0,1 / \Phi\left(x_{0}\right)\right)$ and any $x \in \mathbb{R}$ satisfying

$$
-\frac{\rho_{1}}{\Phi^{-1}(1 / t)} \leqslant x+t \varphi^{\prime}\left(\Phi^{-1}\left(\frac{M}{t}\right)\right) \leqslant \frac{\rho_{2}}{\Phi^{-1}(1 / t)}
$$

we have

$$
p(t, x) \geqslant C \Phi^{-1}\left(\frac{1}{t}\right)
$$

Proof. Without loss of generality, we may assume that $b=0$. By [8, Thm. 5.4], for any $\theta>0$, there is $c>0$ such that for all $t \in\left(0,1 / \Phi\left(x_{0}\right)\right)$ and $|x| \leqslant \theta h^{-1}(1 / t)$,

$$
p\left(t, x+t b_{h^{-1}(1 / t)}\right) \geqslant c\left(h^{-1}\left(\frac{1}{t}\right)\right)^{-1} .
$$

Since by Propositions 6 and 7 we have $h^{-1}(1 / t) \approx 1 / \Phi^{-1}(1 / t)$ for all $t \in\left(0,1 / \Phi\left(x_{0}\right)\right)$, it suffices to prove that

$$
\left|t \varphi^{\prime}\left(\Phi^{-1}\left(\frac{M}{t}\right)\right)+t b_{h^{-1}(1 / t)}\right| \leqslant \frac{c_{1}}{\Phi^{-1}(1 / t)}
$$


for some $c_{1}>0$. To this end, note that for $\lambda_{1}, \lambda_{2}>0$, we have

$$
\begin{aligned}
\left|\varphi^{\prime}\left(\lambda_{1}\right)+b_{\lambda_{2}}\right| & =\left|\int_{0}^{\infty} s\left(\mathbf{1}_{s<\lambda_{2}}-\mathrm{e}^{-\lambda_{1} s}\right) \nu(\mathrm{d} s)\right| \lesssim \lambda_{1} \int_{0}^{\lambda_{2}} s^{2} \nu(\mathrm{d} s)+\int_{\lambda_{2}}^{\infty} s \mathrm{e}^{-\lambda_{1} s} \nu(\mathrm{d} s) \\
& \lesssim \lambda_{1} \lambda_{2}^{2} K\left(\lambda_{2}\right)+\lambda_{1}^{-1} h\left(\lambda_{2}\right) .
\end{aligned}
$$

Now put $\lambda_{1}=\Phi^{-1}(M / t)$ and $\lambda_{2}=h^{-1}(1 / t)$. Then using again Propositions 6 and 7 , we infer that

$$
\left|\varphi^{\prime}\left(\lambda_{1}\right)+b_{\lambda_{2}}\right| \lesssim \frac{1}{t \Phi^{-1}(1 / t)}
$$

and the proof is completed.

Now we treat the right tail of the transition density. In general, based on results concerning various kinds of Lévy processes, we expect the decay to be expressed in terms of Lévy measure. For instance, in the case of unimodal Lévy processes satisfying some scaling conditions, it is known [2, Thm. 21 and Corollary 23] that $p(t, x) \approx p(t, 0) \wedge t \nu(x)$. In the nonsymmetric case, a similar right tail decay is displayed by the transition densities of subordinators (see, e.g., [7]). This is also the case for spectrally one-sided Lévy processes, as the following lemma states. See also Theorem 4 and Remark 6 for comments on two-sided estimate.

Lemma 4. Let $\mathbf{X}$ be a spectrally positive Lévy process of infinite variation with Laplace exponent $\varphi$. Suppose that $\sigma=0$ and $\varphi^{\prime \prime} \in \operatorname{WLSC}\left(\alpha-2, c, x_{0}\right)$ for some $x_{0} \geqslant 0, c \in(0,1]$, and $\alpha>1$. We also assume that the Lévy measure $\nu(\mathrm{d} x)$ has an almost monotone density $\nu(x)$. Then the probability distribution of $\mathbf{X}$ has a density $p(t, \cdot)$. Moreover, there are $\rho_{0}>0$ and $C>0$ such that for all $t \in\left(0,1 / \Phi\left(x_{0} \vee 2 \theta_{1}\right)\right)$ and $x \geqslant \rho_{0} /\left(\Phi^{-1}(1 / t)\right)$, we have

$$
p(t, x) \geqslant C t \nu(x)
$$

Proof. Without loss of generality, we may and do assume that $b=0$. Let $\lambda>0$. We decompose the Lévy measure $\nu(\mathrm{d} x)$ as follows: let $\nu_{1}(\mathrm{~d} x)=\nu_{1}(x) \mathrm{d} x$ and $\nu_{2}(\mathrm{~d} x)=\nu_{2}(x) \mathrm{d} x$, where

$$
\nu_{1}(x)=\frac{1}{2} \nu(x) \mathbf{1}_{[\lambda, \infty)}(x) \quad \text { and } \quad \nu_{2}(x)=\nu(x)-\nu_{1}(x) .
$$

For $u>0$, we set

$$
\begin{gathered}
\varphi_{1}(u)=\int_{0}^{\infty}\left(\mathrm{e}^{-u s}-1\right) \nu_{1}(s) \mathrm{d} s, \\
\varphi_{2}(u)=\int_{0}^{\infty}\left(\mathrm{e}^{-u s}-1+u s \mathbf{1}_{s<1}\right) \nu_{2}(s) \mathrm{d} s+u \int_{0}^{1} s \nu_{1}(s) \mathrm{d} s=\varphi(u)-\varphi_{1}(u) .
\end{gathered}
$$

Let $\mathbf{X}^{(j)}$ be a spectrally positive Lévy processes having the Laplace exponent $\varphi_{j}, j \in\{1,2\}$. First, we note that $\nu / 2 \leqslant \nu_{2} \leqslant \nu$. Thus, for every $u>0$,

$$
\frac{1}{2} \varphi^{\prime \prime}(u) \leqslant \varphi_{2}^{\prime \prime}(u) \leqslant \varphi^{\prime \prime}(u)
$$

and, consequently,

$$
\frac{1}{2} \Phi(u) \leqslant \Phi_{2}(u) \leqslant \Phi(u) .
$$


In particular, since $\varphi^{\prime \prime} \in \mathrm{WLSC}\left(\alpha-2, c, x_{0}\right)$, by Theorem 1 the random variables $X_{t}$ and $X_{t}^{(2)}$ are absolutely continuous for all $t>0$. By $p(t, \cdot)$ and $p^{(2)}(t, \cdot)$ we denote their densities. Note that $\mathbf{X}^{(1)}$ is in fact a compound Poisson process. If we denote its probability distribution by $P_{t}^{(1)}(\mathrm{d} x)$, then by [23, Rem. 27.3]

$$
P_{t}^{(1)}(\mathrm{d} x) \geqslant t \mathrm{e}^{-\nu_{1}(\mathbb{R})} \nu_{1}(x) \mathrm{d} x .
$$

Note that if $\lambda \geqslant c_{1} / \Phi^{-1}(1 / t)$ for some $c_{1}>0$, then by (4.5)

$$
t \nu_{1}(\mathbb{R})=\frac{1}{2} t \int_{\lambda}^{\infty} \nu(x) \mathrm{d} x \leqslant \frac{1}{2} t h\left(\frac{c_{1}}{\Phi^{-1}(1 / t)}\right) \lesssim t h\left(\frac{1}{\Phi^{-1}(1 / t)}\right) \lesssim 1,
$$

where in the penultimate inequality we use [8, Lemma 2.1].

Now denote

$$
x_{t}=-t \varphi_{2}^{\prime}\left(\Phi_{2}^{-1}\left(\frac{M_{0}}{t}\right)\right),
$$

where $M_{0}$ is taken from Lemma 3 . We claim that there is $\rho_{0}>0$ such that for all $t \in\left(0,1 / \Phi\left(x_{0}\right)\right)$,

$$
\frac{\rho_{0}}{\Phi^{-1}(1 / t)} \geqslant-x_{t}
$$

Indeed, note that by (4.14), for any $s>0$,

$$
\Phi_{2}^{-1}(s) \geqslant \Phi^{-1}(s)
$$

Thus, using Proposition 4 and the monotonicity of $\Phi^{-1}$, we conclude that there is $c_{2}>0$ such that

$$
t \varphi_{2}^{\prime}\left(\Phi_{2}^{-1}\left(\frac{M_{0}}{t}\right)\right) \leqslant c_{2} t \frac{M_{0}}{t} \frac{1}{\Phi_{2}^{-1}\left(M_{0} / t\right)} \leqslant \frac{c_{2} M_{0}}{\Phi^{-1}\left(M_{0} / t\right)} \leqslant \frac{c_{2} M_{0}}{\Phi^{-1}(1 / t)}
$$

and (4.17) follows with $\rho_{0}=c_{2} M_{0}$.

Now, we apply Lemma 3 to $\mathbf{X}^{(2)}$. For all $\rho>0$, there is $C>0$ such that for all $t \in\left(0,1 / \Phi_{2}\left(x_{0}\right)\right)$ and $x \in \mathbb{R}$ satisfying

$$
x_{t}-\frac{\rho}{\Phi_{2}^{-1}(1 / t)} \leqslant x \leqslant x_{t}+\frac{\rho}{\Phi_{2}^{-1}(1 / t)},
$$

we have $p^{(2)}(t, x) \geqslant C \Phi_{2}^{-1}(1 / t)$. Note that if $x_{0}>0$, then we may easily extend the above for $t \in$ $\left(0,1 / \Phi\left(x_{0}\right)\right)$. Let $\rho_{0}$ be taken from (4.17) and set $\lambda=x_{t}+\rho / \Phi_{2}^{-1}(1 / t)$, where $\rho=3 \rho_{0} / 2$. Then it follows that $\lambda \geqslant \rho_{0} /\left(2 \Phi_{2}^{-1}(1 / t)\right)$, and, consequently,

$$
\int_{0}^{\lambda} p^{(2)}(t, x) \mathrm{d} x \gtrsim 1
$$

Thus, using (4.15) and (4.16), for $x \geqslant 2 \lambda$, we have

$$
p(t, x)=\int_{\mathbb{R}} p^{(2)}(t, x-y) P_{t}^{(1)}(\mathrm{d} y) \gtrsim t \int_{\mathbb{R}} p^{(2)}(t, x-y) \nu_{1}(y) \mathrm{d} y \geqslant \frac{1}{2} t \int_{\lambda}^{x} p^{(2)}(t, x-y) \nu(y) \mathrm{d} y .
$$


Finally, using the almost monotonicity of $\nu$ and (4.19), we get

$$
p(t, x) \gtrsim t \nu(x) \int_{0}^{\lambda} p^{(2)}(t, y) \mathrm{d} y \gtrsim t \nu(x) .
$$

Now it remains to note that by (4.13), for any $u>0, \varphi_{2}^{\prime}(u) \geqslant \varphi^{\prime}(u)$. Thus by (4.18)

$$
\lambda=-t \varphi_{2}^{\prime}\left(\Phi_{2}^{-1}\left(\frac{M_{0}}{t}\right)\right)+\frac{\rho}{\Phi^{-1}(1 / t)} \leqslant-t \varphi^{\prime}\left(\Phi^{-1}\left(\frac{M_{0}}{t}\right)\right)+\frac{\rho}{\Phi^{-1}(1 / t)} .
$$

The proof is completed.

\section{Sharp two-sided estimates}

This section is devoted to derivation of sharp two-sided estimates. As mentioned in the Introduction, here we will require the upper scaling condition as well to express the Lévy density in terms of Laplace exponent $\varphi$. However, thanks to strict separation from the limit case $\alpha=1$, first, we are able to provide a simpler expression for the localization of $\sup _{x \in \mathbb{R}} p(t, x)$.

Theorem 3. Let $\mathbf{X}$ be a spectrally positive Lévy process of infinite variation with Laplace exponent $\varphi$. Suppose that $\sigma=0$ and $\varphi \in \operatorname{WLSC}\left(\alpha, c, x_{0}\right)$ for some $c \in(0,1], x_{0} \geqslant 0$, and $\alpha>1$. We also assume that $\varphi^{\prime}\left(\theta_{1}\right)=0$. Then for all $-\infty<\chi_{1}<\chi_{2}<\infty$, there is $C>0$ such that for all $t \in\left(0,1 / \Phi\left(x_{0} \vee 2 \theta_{0}\right)\right)$ and $x \in \mathbb{R}$ satisfying

$$
\chi_{1}<x \varphi^{-1}\left(\frac{1}{t}\right)<\chi_{2}
$$

we have

$$
C^{-1} \varphi^{-1}\left(\frac{1}{t}\right) \leqslant p(t, x) \leqslant C \varphi^{-1}\left(\frac{1}{t}\right)
$$

Proof. First, note that by Proposition 8 there is $C^{\prime} \geqslant 1$ such that for all $r \in\left(0,1 / \Phi\left(x_{0} \vee 2 \theta_{0}\right)\right)$,

$$
C^{\prime-1} \Phi^{-1}(r) \leqslant \varphi^{-1}(r) \leqslant C^{\prime} \Phi^{-1}(r) .
$$

Thus, in view of [8, Thm. 3.1] and Propositions 6 and 7, it suffices to prove the first inequality in (5.1). Next, note that the assumptions of Lemma 3 are satisfied. Let $M_{0}$ be taken from Lemma 3 ; for fixed $M>M_{0}$ and $t \in\left(0,1 / \Phi\left(x_{0} \vee 2 \theta_{0}\right)\right)$, we set $x_{t}=-t \varphi^{\prime}\left(\Phi^{-1}(M / t)\right)$. By Propositions 2 and 7 and (5.2) there is $c_{1} \in(0,1]$ such that

$$
t \varphi^{\prime}\left(\Phi^{-1}\left(\frac{M}{t}\right)\right) \geqslant \frac{c_{1}}{\varphi^{-1}(1 / t)} .
$$

Furthermore, by Proposition 4, (4.3), and (5.2) there is $C_{1} \geqslant 1$ such that

$$
t \varphi^{\prime}\left(\Phi^{-1}\left(\frac{M}{t}\right)\right) \leqslant \frac{C_{1}}{\varphi^{-1}(1 / t)} .
$$

Now we apply Lemma 3. Pick $\rho_{1}$ and $\rho_{2}$ so that

$$
-c_{1}-\frac{\rho_{1}}{C^{\prime}} \leqslant \chi_{1} \quad \text { and } \quad-C_{1}+\frac{\rho_{2}}{C^{\prime}} \geqslant \chi_{2} .
$$

Lith. Math. J., 62(1):43-68, 2022. 
Then it is clear that

$$
\left[\frac{\chi_{1}}{\varphi^{-1}(1 / t)}, \frac{\chi_{2}}{\varphi^{-1}(1 / t)}\right] \subset\left(x_{t}-\frac{\rho_{1}}{\Phi^{-1}(1 / t)}, x_{t}+\frac{\rho_{2}}{\Phi^{-1}(1 / t)}\right)
$$

Hence, by Lemma 3 and (5.2), for all $t \in\left(0,1 / \Phi\left(x_{0} \vee 2 \theta_{0}\right)\right)$ and $x \in \mathbb{R}$ satisfying $\chi_{1} \leqslant x \varphi^{-1}(1 / t) \leqslant \chi_{2}$, we have $p(t, x) \gtrsim \varphi^{-1}(1 / t)$, and the theorem follows.

Proceeding exactly as in the proof of [7, Prop. 4.15] and applying Corollary 2, we get the following:

Proposition 9. Assume that the Lévy measure $\nu(\mathrm{d} x)$ has an almost monotone density $\nu(x)$. Suppose that $\varphi^{\prime}\left(\theta_{1}\right)=0$ and $\varphi \in \operatorname{WLSC}\left(\alpha, c, x_{0}\right) \cap \operatorname{WUSC}\left(\beta, C, x_{0}\right)$ for some $c \in(0,1], C \geqslant 1, x_{0} \geqslant \theta_{0}$, and $1<\alpha \leqslant \beta<2$. Then there is $c^{\prime} \in(0,1]$ such that for all $0<x<x_{0}^{-1} \wedge\left(2 \theta_{0}\right)^{-1}$,

$$
\nu(x) \geqslant c^{\prime} x^{-1} \varphi\left(\frac{1}{x}\right)
$$

Now we are ready to prove our main result of this section.

Theorem 4. Let $\mathbf{X}$ be a spectrally positive Lévy process of infinite variation with the Laplace exponent $\varphi$ such that $\theta_{1}=0$ and $\varphi^{\prime}(0)=0$. Suppose that $\sigma=0$ and $\varphi \in \operatorname{WLSC}\left(\alpha, c, x_{0}\right) \cap \operatorname{WUSC}\left(\beta, C, x_{0}\right)$ for some $c \in(0,1], C \geqslant 1, x_{0} \geqslant 0$, and $1<\alpha \leqslant \beta<2$. We also assume that the Lévy measure $\nu(\mathrm{d} x)$ has an almost monotone density $\nu(x)$. Then there is $x_{1} \in(0, \infty]$ such that for all $t \in\left(0,1 / \Phi\left(x_{0}\right)\right)$ and $x \in\left(-\infty, x_{1}\right)$,

$$
p(t, x) \approx \begin{cases}\left(t \varphi^{\prime \prime}(w)\right)^{-1 / 2} \exp \left\{-t\left(w \varphi^{\prime}(w)-\varphi(w)\right)\right\} & \text { if } x \varphi^{-1}\left(\frac{1}{t}\right) \leqslant-1 \\ \varphi^{-1}\left(\frac{1}{t}\right) & \text { if }-1<x \varphi^{-1}\left(\frac{1}{t}\right) \leqslant 1 \\ t x^{-1} \varphi\left(\frac{1}{x}\right) & \text { if } x \varphi^{-1}\left(\frac{1}{t}\right)>1,\end{cases}
$$

where $w=\left(\varphi^{\prime}\right)^{-1}(-x / t)$. If $x_{0}=0$, then $x_{1}=\infty$.

Proof. Set $x_{1}=x_{0}^{-1}$. First, note that in view of Propositions 2 and 4, $\varphi^{\prime \prime} \in \operatorname{WLSC}\left(\alpha-2, c, x_{0}\right)$. Hence, by Corollary 5 , for $\chi_{1}=-M \wedge-1$,

$$
p(t, x) \approx\left(t \varphi^{\prime \prime}(w)\right)^{1 / 2} \exp \left\{-t\left(w \varphi^{\prime}(w)-\varphi(w)\right)\right\}
$$

if only $x \varphi^{-1}(1 / t)<\chi_{1}$. In fact, if $\chi_{1}<-1$, then we also have

$$
\left(t \varphi^{\prime \prime}(w)\right)^{1 / 2} \exp \left\{-t\left(w \varphi^{\prime}(w)-\varphi(w)\right)\right\} \approx \varphi^{-1}\left(\frac{1}{t}\right)
$$

for $\chi_{1} \leqslant x \varphi^{-1}(1 / t) \leqslant-1$. To show this, we first prove the following.

Claim 1. There exist $0<c_{1} \leqslant 1 \leqslant c_{2}$ such that for all $t \in\left(0, c_{1} / \Phi\left(x_{0}\right)\right)$ and $x \in\left(-\infty, x_{1}\right)$ satisfying

$$
\chi_{1} \leqslant x \varphi^{-1}\left(\frac{1}{t}\right) \leqslant-1
$$


we have

$$
-t \varphi^{\prime}\left(\varphi^{-1}\left(\frac{c_{2}}{t}\right)\right) \leqslant x \leqslant-t \varphi^{\prime}\left(\varphi^{-1}\left(\frac{c_{1}}{t}\right)\right) .
$$

Indeed, by Proposition 8 there is $C_{1} \geqslant 1$ such that for all $r>\Phi\left(x_{0}\right)$,

$$
C_{1}^{-1} \Phi^{-1}(r) \leqslant \varphi^{-1}(r) \leqslant C_{1} \Phi^{-1}(r) .
$$

Let $c_{2}=\left(-\chi_{1} C^{\prime} C_{1}^{2}\right)^{\alpha /(\alpha-1)} \in[1, \infty)$, where $C^{\prime}$ is taken from (4.9). Then it follows that

$$
c_{2}^{-1} \varphi^{-1}\left(\frac{c_{2}}{t}\right) \leqslant c_{2}^{(1-\alpha) / \alpha} C_{1}^{2} C^{\prime} \varphi^{-1}\left(\frac{1}{t}\right)=\left(-\chi_{1}\right)^{-1} \varphi^{-1}\left(\frac{1}{t}\right) .
$$

Thus by Proposition 2

$$
x \geqslant-\frac{-\chi_{1}}{\varphi^{-1}(1 / t)} \geqslant-t \frac{\varphi\left(\varphi^{-1}\left(c_{2} / t\right)\right)}{\varphi^{-1}\left(c_{2} / t\right)} \geqslant-t \varphi^{\prime}\left(\varphi^{-1}\left(c_{2} / t\right)\right) .
$$

Moreover, also by Proposition 2 with $c_{1}=C^{-\alpha /(\alpha-1)}$ we have that for $t \in\left(0, c_{1} / \varphi\left(x_{0}\right)\right)$,

$$
t \varphi^{\prime}\left(\varphi^{-1}\left(c_{1} / t\right)\right) \leqslant \frac{C c_{1}}{\varphi^{-1}(1 / t)} \cdot \frac{\varphi^{-1}(1 / t)}{\varphi^{-1}\left(c_{1} / t\right)} \leqslant \frac{C c_{1}^{(\alpha-1) / \alpha}}{\varphi^{-1}(1 / t)}
$$

and thus

$$
x \leqslant-\frac{1}{\varphi^{-1}(1 / t)} \leqslant-t \varphi^{\prime}\left(\varphi^{-1}\left(\frac{c_{1}}{t}\right)\right)
$$

and the claim follows.

Now, using Claim 1, Proposition 7, (4.3), and Proposition 8, we get that for all $t \in\left(0, c_{1} / \Phi\left(x_{0}\right)\right)$,

$$
w \approx \varphi^{-1}\left(\frac{1}{t}\right)
$$

Hence, in view of Proposition 2, $t w \varphi^{\prime}(w) \approx 1$, and, consequently, $\exp \left\{-t\left(w \varphi^{\prime}(w)-\varphi(w)\right)\right\} \approx 1$. Furthermore, by Proposition $4 \varphi^{\prime \prime}(w) \approx w \varphi^{\prime}(w)$, which, combined with (5.5), yields

$$
\frac{1}{\sqrt{t \varphi^{\prime \prime}(w)}} \approx \frac{w}{\sqrt{w \varphi^{\prime}(w)}} \approx \varphi^{-1}\left(\frac{1}{t}\right)
$$

and (5.4) follows for $t \in\left(0, c_{1} / \Phi\left(x_{0}\right)\right)$.

Next, recall that $\theta_{1}=0$ and $\varphi^{\prime}(0)=0$. Therefore, in view of (2.5), we in fact have $b_{r}=-\int_{r}^{\infty} s \nu(s) \mathrm{d} s$.

Now let $x>1 / \varphi^{-1}(1 / t)$. By Theorem 2, Remark 5, and the monotonicity of $\eta$ we have

$$
p(t, x) \lesssim t \eta\left(x-t b_{1 / \Phi^{-1}(1 / t)}\right) \leqslant t \eta(x) .
$$

Thus, by Corollary 2 , for all $t \in\left(0,1 / \Phi\left(x_{0}\right)\right)$ and $x \in\left(0, x_{1}\right)$ such that $x \varphi^{-1}(1 / t)>1$,

$$
p(t, x) \lesssim t x^{-1} \varphi\left(\frac{1}{x}\right) .
$$


Next, by Lemma 4 and Proposition 9 there are $\rho_{0}>0$ and $c>0$ such that for any $t \in\left(0,1 / \Phi\left(x_{0}\right)\right)$ and $x \in\left(0, x_{1}\right)$ satisfying $x \varphi^{-1}(1 / t) \geqslant \rho_{0}$, we have

$$
p(t, x) \geqslant c t x^{-1} \varphi\left(\frac{1}{x}\right) .
$$

Thus if we set $\chi_{2}=1 \vee \rho_{0}$, then, by (5.6) and (5.7), for all $t \in\left(0,1 / \Phi\left(x_{0}\right)\right)$ and $x \in\left(0, x_{1}\right)$ such that $x \varphi^{-1}(1 / t) \geqslant \chi_{2}$,

$$
p(t, x) \approx t x^{-1} \varphi\left(\frac{1}{x}\right)
$$

Finally, by Theorem 3, for all $t \in\left(0,1 / \Phi\left(x_{0}\right)\right)$ and $x \in\left(-\infty, x_{1}\right)$ satisfying $\chi_{1}<x \varphi^{-1}(1 / t)<\chi_{2}$, we have $p(t, x) \approx \varphi^{-1}(1 / t)$. It remains to notice that if $\chi_{2}>1$, then, by the scaling properties of $\varphi$, for all $t \in\left(0,1 / \Phi\left(x_{0}\right)\right)$ and $x \in\left(0, x_{1}\right)$ satisfying $1 \leqslant x \varphi^{-1}(1 / t) \leqslant \chi_{2}$, we have $t x^{-1} \varphi(1 / x) \approx \varphi^{-1}(1 / t)$, which, combined with (5.3), (5.4), and (5.8), finishes the proof for the case $x_{0}=0$. If $x_{0}>0$, then we can use the positivity and continuity to extend the time range from $c_{1} / \Phi\left(x_{0}\right)$ to $1 / \Phi\left(x_{0}\right)$.

Remark 6. Taking into account (4.10), Corollary 2, and Proposition 9, we may see that, in fact,

$$
\nu(x) \approx x^{-1} \varphi\left(\frac{1}{x}\right)
$$

for all $0<x<x_{0}^{-1} \wedge\left(2 \theta_{0}\right)^{-1}$. Therefore by inspecting the proof of Theorem 4 we can show that the term $t x^{-1} \varphi(1 / x)$ in the theorem may be replaced by $t \nu(x)$.

Example 1. Let $\mathbf{X}$ be a spectrally positive $\alpha$-stable process with the Laplace exponent $\varphi(\lambda)=\lambda^{\alpha}$, where $\alpha>1$. Then it is clear that we have $\varphi^{\prime \prime}(\lambda)=\alpha(\alpha-1) \lambda^{\alpha-2}$ and $\left(\varphi^{\prime}\right)^{-1}(y)=(y / \alpha)^{1 /(\alpha-1)}$. Consequently, from Theorem 1 we get that the asymptotics of $p(t, x)$ is of the form

$$
\frac{1}{\sqrt{2 \pi \alpha(\alpha-1)}}\left(\frac{-x}{\alpha}\right)^{(2-\alpha) /(2(\alpha-1))} t^{-1 /(2(\alpha-1))} \exp \left\{-(\alpha-1) t^{-1 /(\alpha-1)}\left(\frac{-x}{\alpha}\right)^{\alpha /(\alpha-1)}\right\}
$$

which, after setting $t=1$, coincides with [26, Thm. 2.5.2]. Moreover, by Theorem 4

$$
p(t, x) \approx \begin{cases}\frac{1}{\sqrt{2 \pi \alpha(\alpha-1)}}\left(\frac{-x}{\alpha}\right)^{(2-\alpha) /(2(\alpha-1))} t^{-1 /(2(\alpha-1))} & \\ \times \exp \left\{-(\alpha-1) t^{-1 /(\alpha-1)}\left(\frac{-x}{\alpha}\right)^{\alpha /(\alpha-1)}\right\} & \text { if } \frac{x}{t^{1 / \alpha}} \leqslant-1 \\ t^{-1 / \alpha} & \text { if }-1<\frac{x}{t^{1 / \alpha}} \leqslant 1 \\ \frac{t}{x^{1+\alpha}} & \text { if } \frac{x}{t^{1 / \alpha}}>1 .\end{cases}
$$

For $\alpha=1$, in view of [22, Prop. 1.2.12], we have $\varphi(\lambda)=\lambda \ln \lambda$. Therefore $\varphi^{\prime \prime}(\lambda)=\lambda^{-1}$ and $\left(\varphi^{\prime}\right)^{-1}(y)=$ $\mathrm{e}^{y-1}$. By Theorem 1 we get the following form of the asymptotics:

$$
\frac{1}{\sqrt{2 \pi t}} \exp \left\{-\frac{-x / t-1}{2}-t \mathrm{e}^{-x / t-1}\right\}
$$

which again, after substituting $t=1$, coincides with [26, Thm. 2.5.2]. Unfortunately, Theorem 4 cannot be applied due to the scaling condition with $\alpha=1$ only. Also, note that for the case of Brownian motion, using Theorem 1 it is straightforward to obtain a Gaussian density in the asymptotics. 
Lastly, let us justify Remark 1 by constructing an example of spectrally positive Lévy process of unbounded variation for which the lower scaling property holds only with $\alpha<1$.

Example 2. Let us consider the measure $\nu(\mathrm{d} x)$ with density defined as follows: for $x \in(0,1 / 2]$, set

$$
\nu(x)= \begin{cases}c_{k} x^{-5 / 2}, & x \in\left[((2 k+1) !)^{-1},((2 k) !)^{-1}\right], \\ c_{k} \sqrt{(2 k+1) !} x^{-3 / 2}, & x \in\left[((2 k+2) !)^{-1},((2 k+1) !)^{-1}\right],\end{cases}
$$

where $c_{k}=((2 k) ! !)^{-1 / 2}$. For $x>1 / 2$, we put $\nu(x)=0$. As in the proof of [7, Prop. 3.8] and [7, Prop. 4.15], we conclude that

$$
\varphi^{\prime \prime}(x) \approx x^{-3} \nu\left(\frac{1}{x}\right) \approx \begin{cases}c_{k} x^{-1 / 2}, & x \in[(2 k) !,(2 k+1) !], \\ c_{k} \sqrt{(2 k+1) !} x^{-3 / 2}, & x \in[(2 k+1) !,(2 k+2) !] .\end{cases}
$$

By construction, $\varphi^{\prime \prime}$ has the lower and upper Matuszewska indices equal to $-3 / 2$ and $-1 / 2$, respectively, and, consequently, the lower scaling property holds only with $\alpha<1$.

Now let us define the Lévy process $\mathbf{X}$ by setting $\sigma=0, b=0$, and $\nu$ as in (5.9). Then we have

$$
\begin{aligned}
\int_{0}^{1} x \nu(x) \mathrm{d} x & \geqslant \sum_{k=1}^{\infty} c_{k} \int_{1 /(2 k+1) !}^{1 /(2 k) !} x^{-3 / 2} \mathrm{~d} x=2 \sum_{k=1}^{\infty} c_{k}(\sqrt{(2 k+1) !}-\sqrt{(2 k) !}) \\
& \geqslant \frac{2}{3} \sum_{k=1}^{\infty} c_{k} \sqrt{(2 k+1) !}=\infty .
\end{aligned}
$$

Therefore $\mathbf{X}$ is of unbounded variation.

Acknowledgment. I would like to express my deep gratitude to Tomasz Grzywny for helpful discussions, numerous valuable remarks, and for reading the first version of the manuscript.

Open Access. This article is licensed under a Creative Commons Attribution 4.0 International License, which permits use, sharing, adaptation, distribution and reproduction in any medium or format, as long as you give appropriate credit to the original author(s) and the source, provide a link to the Creative Commons licence, and indicate if changes were made. The images or other third party material in this article are included in the article's Creative Commons licence, unless indicated otherwise in a credit line to the material. If material is not included in the article's Creative Commons licence and your intended use is not permitted by statutory regulation or exceeds the permitted use, you will need to obtain permission directly from the copyright holder. To view a copy of this licence, visit http: / / creativecommons.org/licenses/by/4 . 0 /

\section{References}

1. J. Bertoin, Lévy Processes, Camb. Tracts Math., Vol. 121, Cambridge Univ. Press, Cambridge, 1996.

2. K. Bogdan, T. Grzywny, and M. Ryznar, Density and tails of unimodal convolution semigroups, J. Funct. Anal., 266(6):3543-3571, 2014.

3. Z.-Q. Chen, P. Kim, T. Kumagai, and J. Wang, Time fractional Poisson equations: Representations and estimates, J. Funct. Anal., 278(2):108311, 2020.

4. S. Cho and P. Kim, Estimates on transition densities of subordinators with jumping density decaying in mixed polynomial orders, Stochastic Processes Appl., 139:229-279, 2021. 
5. D.J. Emery, Exit problem for a spectrally positive process, Adv. Appl. Probab., 5:498-520, 1973.

6. T. Grzywny, On Harnack inequality and Hölder regularity for isotropic unimodal Lévy processes, Potential Anal., 41(1):1-29, 2014.

7. T. Grzywny, Ł. Leżaj, and B. Trojan, Transition densities of subordinators of positive order, J. Inst. Math. Jussieu, to appear.

8. T. Grzywny and K. Szczypkowski, Lévy processes: Concentration function and heat kernel bounds, Bernoulli, 26(4): 3191-3223, 2020.

9. T. Grzywny and K. Szczypkowski, Estimates of heat kernel for non-symmetric Lévy processes, Forum Math., 33(5): 1207-1236, 2021.

10. F. Hubalek and E. Kyprianou, Old and new examples of scale functions for spectrally negative Lévy processes, in Seminar on Stochastic Analysis, Random Fields and Applications VI, Prog. Probab., Vol. 63, Birkhäuser, Basel, 2011, pp. 119-145.

11. N. Jacob, Pseudo Differential Operators and Markov Processes. Vol. I, Imperial College Press, London, 2001.

12. K. Kaleta and P. Sztonyk, Estimates of transition densities and their derivatives for jump Lévy processes, J. Math. Anal. Appl., 431(1):260-282, 2015.

13. K. Kaleta and P. Sztonyk, Small-time sharp bounds for kernels of convolution semigroups, J. Anal. Math., 132(1): 355-394, 2017.

14. V. Knopova, Compound kernel estimates for the transition probability density of a Lévy process in $\mathbb{R}^{n}$, Teor. Ĭmovirn. Mat. Stat., 89:51-63, 2013.

15. V. Knopova and A. Kulik, Intrinsic small time estimates for distribution densities of Lévy processes, Random Oper. Stoch. Equ., 21(4):321-344, 2013.

16. A. Kuznetsov, A.E. Kyprianou, and V. Rivero, The theory of scale functions for spectrally negative Lévy processes, in Lévy Matters II, Lect. Notes Math., Vol. 2061, pp. 97-186, Springer, Heidelberg, 2012.

17. A.E. Kyprianou, Introductory Lectures on Fluctuations of Lévy Processes with Applications, Universitext, Springer, Berlin, 2006.

18. P. Patie and A. Vaidyanathan, The log-Lévy moment problem via Berg-Urbanik semigroups, Stud. Math., 253(3): 219-257, 2020.

19. J. Picard, Density in small time for Lévy processes, ESAIM, Probab. Stat., 1:357-389, 1997.

20. W.E. Pruitt, The growth of random walks and Lévy processes, Ann. Probab., 9(6):948-956, 1981.

21. L.C.G. Rogers, The two-sided exit problem for spectrally positive Lévy processes, Adv. Appl. Probab., 22(2):486487, 1990.

22. G. Samorodnitsky and M.S. Taqqu, Stable non-Gaussian Random Processes: Stochastic Models with Infinite Variance, Stochastic Modeling, Chapman \& Hall, New York, 1994.

23. K.-I. Sato, Lévy Processes and Infinitely Divisible Distributions, Camb. Stud. Adv. Math., Vol. 68, Cambridge Univ. Press, Cambridge, 1999.

24. R.L. Schilling, R. Song, and Z. Vondraček, Bernstein Functions: Theory and Applications, 2nd ed., De Gruyter Stud. Math., Vol. 37, Walter de Gruyter, Berlin, 2012.

25. L. Takács, Combinatorial Methods in the Theory of Stochastic Processes, John Wiley \& Sons, New York, London, Sydney, 1967.

26. V.M. Zolotarev, The first passage time of a level and the behaviour at infinity for a class of processes with independent increments, Theory Probab. Appl., 9(4):653-662, 1964.

27. V.M. Zolotarev, One-Dimensional Stable Distributions, Transl. Math. Monogr., Vol. 65, AMS, Providence, RI, 1986. 